\title{
Approximation Algorithms for Computing Maximin Share Allocations
}

GEORGIOS AMANATIDIS, Athens University of Economics and Business

EVANGELOS MARKAKIS, Athens University of Economics and Business

AFSHIN NIKZAD, Stanford University

AMIN SABERI, Stanford University

\begin{abstract}
We study the problem of computing maximin share allocations, a recently introduced fairness notion. Given a set of $n$ agents and a set of goods, the maximin share of an agent is the best she can guarantee to herself, if she is allowed to partition the goods in any way she prefers, into $n$ bundles, and then receive her least desirable bundle. The objective then is to find a partition, where each agent is guaranteed her maximin share. Such allocations do not always exist, hence we resort to approximation algorithms. Our main result is a $2 / 3$ approximation, that runs in polynomial time for any number of agents and goods. This improves upon the algorithm of Procaccia and Wang [2014], which is also a 2/3-approximation but runs in polynomial time only for a constant number of agents. To achieve this, we redesign certain parts of the algorithm in Procaccia and Wang [2014], exploiting the construction of carefully selected matchings in a bipartite graph representation of the problem. Furthermore, motivated by the apparent difficulty in establishing lower bounds, we undertake a probabilistic analysis. We prove that in randomly generated instances, maximin share allocations exist with high probability. This can be seen as a justification of previously reported experimental evidence. Finally, we provide further positive results for two special cases arising from previous works. The first is the intriguing case of 3 agents, where we provide an improved 7/8-approximation. The second case is when all item values belong to $\{0,1,2\}$, where we obtain an exact algorithm.
\end{abstract}

CCS Concepts: • Theory of computation $\rightarrow$ Approximation algorithms analysis; Algorithmic game theory; $\bullet$ Mathematics of computing $\rightarrow$ Combinatorial algorithms;

Additional Key Words and Phrases: Fair Division, Maximin Share

ACM Reference Format:

Georgios Amanatidis, Evangelos Markakis, Afshin Nikzad, and Amin Saberi, 2017. Approximation Algorithms for Computing Maximin Share Allocations. ACM Trans. Algor. V, N, Article A (January YYYY), 30 pages.

DOI : http://dx.doi.org/10.1145/0000000.0000000

\section{INTRODUCTION}

We study a recently proposed fair division problem in the context of allocating indivisible goods. Fair division has attracted the attention of various scientific disciplines, including among others, mathematics, economics, and political science. Ever since the first attempt for a formal treatment by Steinhaus, Banach, and Knaster [Steinhaus 1948], many interesting and challenging questions have emerged. Over the past deca-

A preliminary version of this work has appeared in Amanatidis et al. [2015].

Research co-financed by the European Union (European Social Fund - ESF) and Greek national funds through the Operational Program "Education and Lifelong Learning" of the National Strategic Reference Framework (NSRF) - Research Funding Program: "THALES - Investing in knowledge society through the European Social Fund".

Author's addresses: G. Amanatidis and E. Markakis, Department of Informatics, Athens University of Economics and Business, Athens, Greece; A. Nikzad and A. Saberi, Department of Management Science and Engineering, Stanford University, CA, USA.

Permission to make digital or hard copies of all or part of this work for personal or classroom use is granted without fee provided that copies are not made or distributed for profit or commercial advantage and that copies bear this notice and the full citation on the first page. Copyrights for components of this work owned by others than ACM must be honored. Abstracting with credit is permitted. To copy otherwise, or republish, to post on servers or to redistribute to lists, requires prior specific permission and/or a fee. Request permissions from permissions@acm.org.

(C) YYYY ACM. 1549-6325/YYYY/01-ARTA $\$ 15.00$

DOI : http://dx.doi.org/10.1145/0000000.0000000 
des, a vast literature has developed, see e.g., [Brams and Taylor 1996; Robertson and Webb 1998], and several notions of fairness have been suggested. The area gradually gained popularity in computer science as well, as most of the questions are inherently algorithmic, see [Even and Paz 1984; Edmonds and Pruhs 2006; Woeginger and Sgall 2007], among others, for earlier works and the surveys by Procaccia [2015] and by Bouveret et al. [2016] on more recent results.

The objective in fair division problems is to allocate a set of resources to a set of $n$ agents in a way that leaves every agent satisfied. In the continuous case, the available resources are typically represented by the interval $[0,1]$, whereas in the discrete case, we have a set of distinct, indivisible goods. The preferences of each agent are represented by a valuation function, which is usually an additive function (additive on the set of goods in the discrete case, or a probability distribution on $[0,1]$ in the continuous case). Given such a setup, many solution concepts have been proposed as to what constitutes a fair solution. Some of the standard ones include proportionality, envy-freeness, equitability and several variants of them. The most related concept to our work is proportionality, where an allocation is called proportional, if each agent receives a bundle of goods that is worth at least $1 / n$ of the total value according to her valuation function.

Interestingly, all the above mentioned solutions and several others can be attained in the continuous case. Apart from mere existence, in some cases we can also have efficient algorithms, see e.g., [Even and Paz 1984] for proportionality and [Aziz and MacKenzie 2016] for some recent progress on envy-freeness. In the presence of indivisible goods however, the picture is quite different. We cannot guarantee existence and it is even NP-hard to decide whether a given instance admits fair allocations. In fact, in most cases it is hard to produce decent approximation guarantees.

Motivated by the question of what can we guarantee in the discrete case, we focus on a concept recently introduced by Budish [2011], that can be seen as a relaxation of proportionality. The rationale is as follows: suppose that an agent, say agent $i$, is asked to partition the goods into $n$ bundles and then the rest of the agents choose a bundle before $i$. In the worst case, agent $i$ will be left with her least valuable bundle. Hence, a risk-averse agent would choose a partition that maximizes the minimum value of a bundle in the partition. This value is called the maximin share of agent $i$. The objective then is to find an allocation where every agent receives at least her maximin share. Even for this notion, existence is not guaranteed under indivisible goods [Procaccia and Wang 2014; Kurokawa et al. 2016], despite the encouraging experimental evidence [Bouveret and Lemaître 2016; Procaccia and Wang 2014]. However, it is possible to have constant factor approximations, as has been recently shown [Procaccia and Wang 2014] (see also our related work section).

Contribution: Our main result, in Section 4 , is a $(2 / 3-\varepsilon)$-approximation algorithm, for any constant $\varepsilon>0$, that runs in polynomial time for any number of agents and any number of goods. That is, the algorithm produces an allocation where every agent receives a bundle worth at least $2 / 3-\varepsilon$ of her maximin share. Our result improves upon the 2/3-approximation of Procaccia and Wang [2014], which runs in polynomial time only for a constant number of agents. To achieve this, we redesign certain parts of their algorithm, arguing about the existence of appropriate, carefully constructed matchings in a bipartite graph representation of the problem. Before that, in Section 3 , we provide a much simpler and faster $1 / 2$-approximation algorithm. Despite the worse factor, this algorithm still has its own merit due to its simplicity.

Moreover, we study two special cases, motivated by previous works. The first one is the case of $n=3$ agents. This is an interesting turning point on the approximability of the problem; for $n=2$, there always exist maximin share allocations, but adding a 
third agent makes the problem significantly more complex, and the best known ratio was $3 / 4$ [Procaccia and Wang 2014]. We provide an algorithm with an approximation guarantee of $7 / 8$, by examining more deeply the set of allowed matchings that we can use to satisfy the agents. The second case is the setting where all item values belong to $\{0,1,2\}$. This is an extension of the $\{0,1\}$ setting studied by Bouveret and Lemaitre [2016] and we show that there always exists a maximin share allocation, for any number of agents.

Finally, motivated by the apparent difficulty in finding impossibility results on the approximability of the problem, we undertake a probabilistic analysis in Section 6. Our analysis shows that in randomly generated instances, maximin share allocations exist with high probability. This may be seen as a justification of the reported experimental evidence [Bouveret and Lemaître 2016; Procaccia and Wang 2014], which show that maximin share allocations exist in most cases.

\subsection{Related Work}

For an overview of the classic fairness notions and related results, we refer the reader to the books of Brams and Taylor [1996], and Robertson and Webb [1998]. The notion we study here was introduced by Budish [2011] for ordinal utilities (i.e., agents have rankings over alternatives), building on concepts by Moulin [1990]. Later on, Bouveret and Lemaitre [2016] defined the notion for cardinal utilities, in the form that we study it here, and provided many important insights as well as experimental evidence. The first constant factor approximation algorithm was given by Procaccia and Wang [2014], achieving a 2/3-approximation but in time exponential in the number of agents.

On the negative side, constructions of instances where no maximin share allocation exists, even for $n=3$, have been provided both by Procaccia and Wang [2014], and by Kurokawa et al. [2016]. These elaborate constructions, along with the extensive experimentation of Bouveret and Lemaître [2016], reveal that it has been challenging to produce better lower bounds, i.e., instances where no $\alpha$-approximation of a maximin share allocation exists, even for $\alpha$ very close to 1 . Driven by these observations, a probabilistic analysis, similar in spirit but more general than ours, is carried out by Kurokawa et al. [2016]. In our analysis in Section 6, all values are uniformly drawn from $[0,1]$; Kurokawa et al. [2016] show a similar result with ours but for a a wide range of distributions over $[0,1]$, establishing that maximin share allocations exist with high probability under all such distributions. However, their analysis, general as it may be, needs very large values of $n$ to guarantee relatively high probability, hence it does not fully justify the experimental results discussed above.

Recently, some variants of the problem have also been considered. Barman and Murthy [2017] gave a constant factor approximation of $1 / 10$ for the case where the agents have submodular valuation functions. It remains an interesting open problem to determine whether better factors are achievable for submodular, or other nonadditive functions. Along a different direction, Caragiannis et al. [2016] introduced the notion of pairwise maximin share guarantee and provided approximation algorithms. Although conceptually this is not too far apart from maximin shares, the two notions are incomparable.

Another aspect that has been studied is the design of truthful mechanisms providing approximate maximin share fairness guarantees. Note that our work here does not deal with incentive issues. Looking at this as a mechanism design problem without money, Amanatidis et al. [2016] provide both positive and negative results exhibiting a clear separation between what can be achieved with and without the truthfulness constraint. Even further, Amanatidis et al. [2017] completely characterized truthful mechanisms for two agents, which in turn implied tight bounds on the approximability of maximin share fairness by truthful mechanisms. 
Finally, a seemingly related problem is that of max-min fairness (also known as the Santa Claus problem) [Asadpour and Saberi 2007; Bansal and Sviridenko 2006; Bezakova and Dani 2005]. In this problem we want to find an allocation where the value of the least happy person is maximized. With identical agents, this coincides with our problem, but beyond this special case the two problems exhibit very different behavior.

\section{DEFINITIONS AND NOTATION}

For any $k \in \mathbb{N}$, we denote by $[k]$ the set $\{1, \ldots, k\}$. Let $N=[n]$ be a set of $n$ agents and $M=[m]$ be a set of indivisible items. Following the usual setup in the fair division literature, we assume each agent has an additive valuation function $v_{i}(\cdot)$, so that for every $S \subseteq M, v_{i}(S)=\sum_{j \in S} v_{i}(\{j\})$. For $j \in M$, we will use $v_{i j}$ instead of $v_{i}(\{j\})$.

Given any subset $S \subseteq M$, an allocation of $S$ to the $n$ agents is a partition $T=$ $\left(T_{1}, \ldots, T_{n}\right)$, where $T_{i} \cap T_{j}=\emptyset$ and $\bigcup T_{i}=S$. Let $\Pi_{n}(S)$ be the set of all partitions of a set $S$ into $n$ bundles.

Definition 2.1. Given a set of $n$ agents, and any set $S \subseteq M$, the $n$-maximin share of an agent $i$ with respect to $S$, is:

$$
\boldsymbol{\mu}_{i}(n, S)=\max _{T \in \Pi_{n}(S)} \min _{T_{j} \in T} v_{i}\left(T_{j}\right) .
$$

Note that $\boldsymbol{\mu}_{i}(n, S)$ depends on the valuation function $v_{i}(\cdot)$ but is independent of any other function $v_{j}(\cdot)$ for $j \neq i$. When $S=M$, we refer to $\boldsymbol{\mu}_{i}(n, M)$ as the maximin share of agent $i$. The solution concept we study asks for a partition that gives each agent her maximin share.

Definition 2.2. Given a set of agents $N$, and a set of goods $M$, a partition $T=$ $\left(T_{1}, \ldots, T_{n}\right) \in \Pi_{n}(M)$ is called a maximin share (MMS) allocation if $v_{i}\left(T_{i}\right) \geq \boldsymbol{\mu}_{i}(n, M)$, for every agent $i \in N$.

Before we continue, a few words are in order regarding the appeal of this new concept. First of all, it is very easy to see that having a maximin share guarantee to every agent forms a relaxation of proportionality, see Claim 3.1. Given the known impossibility results for proportional allocations under indivisible items, it is worth investigating whether such relaxations are easier to attain. Second, the maximin share guarantee has an intuitive interpretation; for an agent $i$, it is the value that could be achieved if we run the generalization of the cut-and-choose protocol for multiple agents, with $i$ being the cutter. In other words, it is the value that agent $i$ can guarantee to himself, if he were given the advantage to control the partition of the items into bundles, but not the allocation of the bundles to the agents.

Example 2.3. Consider an instance with three agents and five items:

\begin{tabular}{cccccc} 
& $a$ & $b$ & $c$ & $d$ & $e$ \\
\hline Agent 1 & $1 / 2$ & $1 / 2$ & $1 / 3$ & $1 / 3$ & $1 / 3$ \\
Agent 2 & $1 / 2$ & $1 / 4$ & $1 / 4$ & $1 / 4$ & 0 \\
Agent 3 & $1 / 2$ & $1 / 2$ & 1 & $1 / 2$ & $1 / 2$ \\
\hline
\end{tabular}

If $M=\{a, b, c, d, e\}$ is the set of items, one can see that $\boldsymbol{\mu}_{1}(3, M)=1 / 2, \boldsymbol{\mu}_{2}(3, M)=1 / 4$, $\boldsymbol{\mu}_{3}(3, M)=1$. E.g., for agent 1, no matter how she partitions the items into three bundles, the worst bundle will be worth at most $1 / 2$ for her, and she achieves this with the partition $(\{a\},\{b, c\},\{d, e\})$. Similarly, agent 3 can guarantee a value of 1 (which is best possible as it is equal to $\left.v_{3}(M) / n\right)$ by the partition $(\{a, b\},\{c\},\{d, e\})$. 
Note that this instance admits a maximin share allocation, e.g., $(\{a\},\{b, c\},\{d, e\})$, and in fact this is not unique. Note also that if we remove some agent, say agent 2 , the maximin values for the other two agents increase. E.g., $\boldsymbol{\mu}_{1}(2, M)=1$, achieved by the partition $(\{a, b\},\{c, d, e\})$. Similarly, $\boldsymbol{\mu}_{3}(2, M)=3 / 2$.

As shown in [Procaccia and Wang 2014], maximin share allocations do not always exist. Hence, our focus is on approximation algorithms, i.e., on algorithms that produce a partition where each agent $i$ receives a bundle worth (according to $v_{i}$ ) at least $\rho$. $\boldsymbol{\mu}_{i}(n, M)$, for some $\rho \leq 1$.

\section{WARMUP: SOME USEFUL PROPERTIES AND A POLYNOMIAL TIME 1/2-APPROXIMATION}

We find it instructive to provide first a simpler and faster algorithm that achieves a worse approximation of $1 / 2$. In the course of obtaining this algorithm, we also identify some important properties and insights that we will use in the next sections.

We start with an upper bound on our solution for each agent. The maximin share guarantee is a relaxation of proportionality, so we trivially have:

Claim 3.1. For every $i \in N$ and every $S \subseteq M, \boldsymbol{\mu}_{i}(n, S) \leq \frac{v_{i}(S)}{n}=\frac{\sum_{j \in S} v_{i j}}{n}$.

PROOF. This follows by the definition of maximin share. If there existed a partition where the minimum value for agent $i$ exceeded the above bound, then the total value for agent $i$ would be more than $\sum_{j \in S} v_{i j}$.

Based on this, we now show how to get an additive approximation. Algorithm 1 below achieves an additive approximation of $v_{\max }$, where $v_{\max }=\max _{i, j} v_{i j}$. This simple algorithm, which we will refer to as the Greedy Round-Robin Algorithm, has also been discussed by Bouveret and Lemaître [2016], where it was shown that when all item values are in $\{0,1\}$, it produces an exact maximin share allocation. At the same time, we note that the algorithm also achieves envy-freeness up to one item, another solution concept defined by Budish [2011], and further discussed in Caragiannis et al. [2016]. Finally, some variations of this algorithm have also been used in other allocation problems, see e.g., Brams and King [2005], or the protocol in Bouveret and Lang [2011]. We discuss further the properties of Greedy Round-Robin in Section 6.

In the statement of the algorithm below, the set $V_{N}$ is the set of valuation functions $V_{N}=\left\{v_{i}: i \in N\right\}$, which can be encoded as a valuation matrix since the functions are additive.

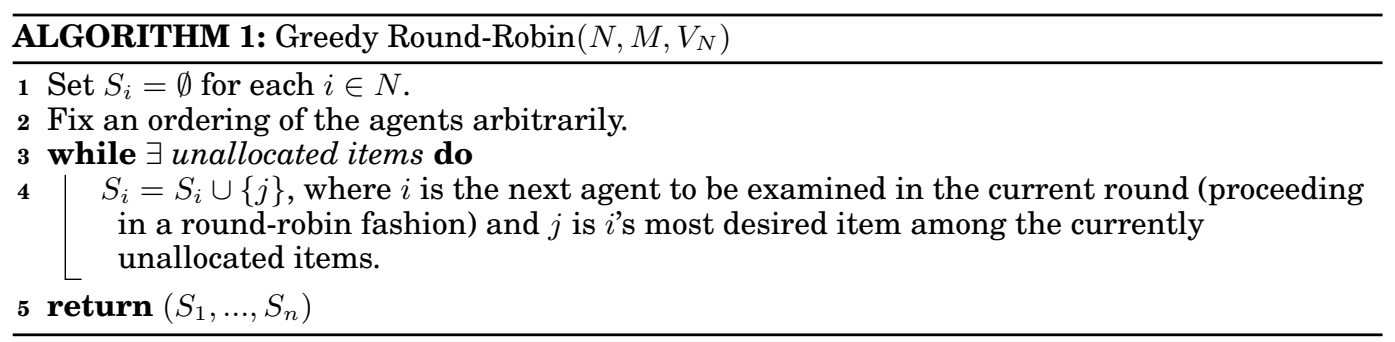

THEOREM 3.2. If $\left(S_{1}, \ldots, S_{n}\right)$ is the output of Algorithm 1, then for every $i \in N$,

$$
v_{i}\left(S_{i}\right) \geq \frac{\sum_{j \in M} v_{i j}}{n}-v_{\max } \geq \boldsymbol{\mu}_{i}(n, M)-v_{\max } .
$$

Proof. Let $\left(S_{1}, \ldots, S_{n}\right)$ be the output of Algorithm 1. We first prove the following claim about the envy of each agent towards the rest of the agents: 
Claim 3.3. For every $i, j \in N, v_{i}\left(S_{i}\right) \geq v_{i}\left(S_{j}\right)-v_{\max }$.

Proof. Fix an agent $i$, and let $j \neq i$. We will upper bound the difference $v_{i}\left(S_{j}\right)-$ $v_{i}\left(S_{i}\right)$. If $j$ comes after $i$ in the order chosen by the algorithm, then the statement of the claim trivially holds, since $i$ always picks an item at least as desirable as the one $j$ picks. Suppose that $j$ precedes $i$ in the ordering. The algorithm proceeds in $\ell=\lceil m / n\rceil$ rounds. In each round $k$, let $r_{k}$ and $r_{k}^{\prime}$ be the items allocated to $j$ and $i$ respectively. Then

$$
v_{i}\left(S_{j}\right)-v_{i}\left(S_{i}\right)=\left(v_{i, r_{1}}-v_{i, r_{1}^{\prime}}\right)+\left(v_{i, r_{2}}-v_{i, r_{2}^{\prime}}\right)+\cdots+\left(v_{i, r_{\ell}}-v_{i, r_{\ell}^{\prime}}\right) .
$$

Note that there may be no item $r_{\ell}^{\prime}$ in the last round if the algorithm runs out of goods but this does not affect the analysis (simply set $v_{i, r_{\ell}^{\prime}}=0$ ).

Since agent $i$ picks her most desirable item when it is her turn to choose, this means that for two consecutive rounds $k$ and $k+1$ it holds that $v_{i, r_{k}^{\prime}} \geq v_{i, r_{k+1}}$. This directly implies that

$$
v_{i}\left(S_{j}\right)-v_{i}\left(S_{i}\right) \leq v_{i, r_{1}}-v_{i, r_{\ell}^{\prime}} \leq v_{i, r_{1}} \leq v_{\max } .
$$

If we now sum up the statement of Claim 3.3 for each $j$, we get: $n v_{i}\left(S_{i}\right) \geq \sum_{j} v_{i}\left(S_{j}\right)-$ $n v_{\max }$, which implies

$$
v_{i}\left(S_{i}\right) \geq \frac{\sum_{j} v_{i}\left(S_{j}\right)}{n}-v_{\max }=\frac{\sum_{j \in M} v_{i j}}{n}-v_{\max } \geq \boldsymbol{\mu}_{i}(n, M)-v_{\max },
$$

where the last inequality holds by Claim 3.1.

The next important ingredient is the following monotonicity property, which says that we can allocate a single good to an agent without decreasing the maximin share of other agents. Note that this lemma also follows from Lemma 1 of Bouveret and Lemaitre [2016], yet, for completeness, we prove it here as well.

LEMMA 3.4 (MONOTONICITY PROPERTY). For any agent $i$ and any good $j$, it holds that

$$
\boldsymbol{\mu}_{i}(n-1, M \backslash\{j\}) \geq \boldsymbol{\mu}_{i}(n, M) .
$$

Proof. Let us look at agent $i$, and consider a partition of $M$ that attains her maximin share. Let $\left(S_{1}, \ldots, S_{n}\right)$ be this partition. Without loss of generality, suppose $j \in S_{1}$. Consider the remaining partition $\left(S_{2}, \ldots, S_{n}\right)$ enhanced in an arbitrary way by the items of $S_{1} \backslash\{j\}$. This is a $(n-1)$-partition of $M \backslash\{j\}$ where the value of agent $i$ for any bundle is at least $\boldsymbol{\mu}_{i}(n, M)$. Thus, we have $\boldsymbol{\mu}_{i}(n-1, M \backslash\{j\}) \geq \boldsymbol{\mu}_{i}(n, M)$.

We are now ready for the 1/2-approximation, obtained by Algorithm 2 below, which is based on using Greedy Round-Robin, but only after we allocate first the most valuable goods. This is done so that the value of $v_{\max }$ drops to an extent that Greedy RoundRobin can achieve a multiplicative approximation. 


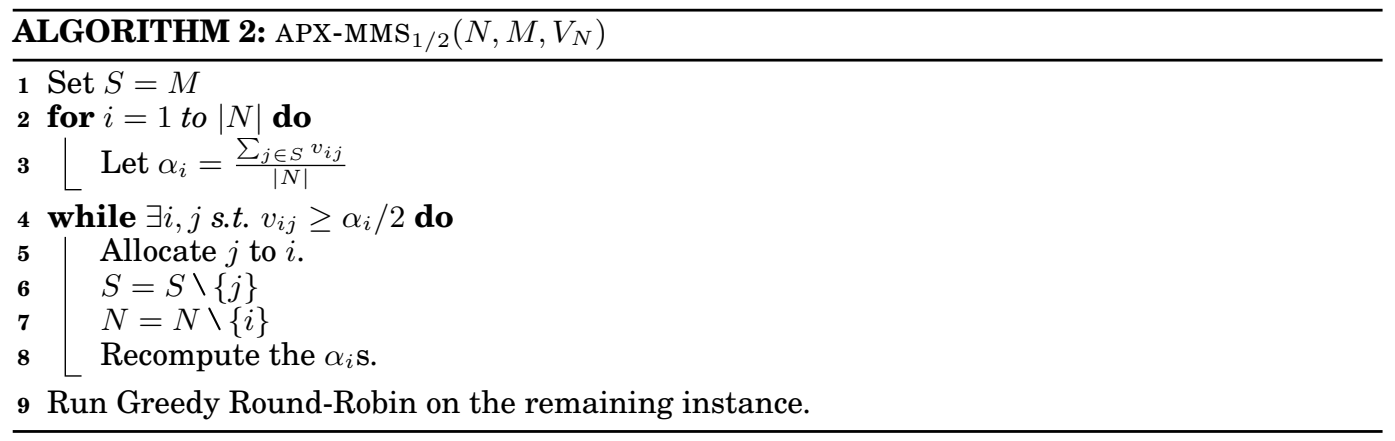

THEOREM 3.5. Let $N$ be a set of $n$ agents, and let $M$ be a set of goods. Algorithm 2 produces an allocation $\left(S_{1}, \ldots, S_{n}\right)$ such that

$$
v_{i}\left(S_{i}\right) \geq \frac{1}{2} \boldsymbol{\mu}_{i}(n, M), \forall i \in N .
$$

Proof. We will distinguish two cases. Consider an agent $i$ who was allocated a single item during the first phase of the algorithm (lines 4 - 8). Suppose that at the time when $i$ was given her item, there were $n_{1}$ active agents, $n_{1} \leq n$, and that $S$ was the set of currently unallocated items. By the design of the algorithm, this means that the value of what $i$ received is at least

$$
\frac{\sum_{j \in S} v_{i j}}{2 n_{1}} \geq \frac{1}{2} \boldsymbol{\mu}_{i}\left(n_{1}, S\right)
$$

where the inequality follows by Claim 3.1. But now if we apply the monotonicity property (Lemma 3.4) $n-n_{1}$ times, we get that $\boldsymbol{\mu}_{i}\left(n_{1}, S\right) \geq \boldsymbol{\mu}_{i}(n, M)$, and we are done.

Consider now an agent $i$, who gets a bundle of goods according to Greedy RoundRobin, in the second phase of the algorithm. Let $n_{2}$ be the number of active agents at that point, and $S$ be the set of goods that are unallocated before Greedy Round-Robin is executed. We know that $v_{\max }$ at that point is less than half the current value of $\alpha_{i}$ for agent $i$. Hence by the additive guarantee of Greedy Round-Robin, we have that the bundle received by agent $i$ has value at least

$$
\frac{\sum_{j \in S} v_{i j}}{n_{2}}-v_{\max }>\frac{\sum_{j \in S} v_{i j}}{n_{2}}-\frac{\alpha_{i}}{2}=\frac{\sum_{j \in S} v_{i j}}{2 n_{2}} \geq \frac{1}{2} \boldsymbol{\mu}_{i}\left(n_{2}, S\right) .
$$

Again, after applying the monotonicity property repeatedly, we get that $\boldsymbol{\mu}_{i}\left(n_{2}, S\right) \geq$ $\boldsymbol{\mu}_{i}(n, M)$, which completes the proof.

\section{A POLYNOMIAL TIME $\left(\frac{2}{3}-\varepsilon\right)$-APPROXIMATION}

The main result of this section is Theorem 4.1, establishing a polynomial time algorithm for achieving a 2/3-approximation to the maximin share of each agent.

THEOREM 4.1. Let $N$ be a set of $n$ agents, and let $M$ be a set of goods. For any constant $\varepsilon>0$, Algorithm 3 produces in polynomial time an allocation $\left(S_{1}, \ldots, S_{n}\right)$, such that

$$
v_{i}\left(S_{i}\right) \geq\left(\frac{2}{3}-\varepsilon\right) \boldsymbol{\mu}_{i}(n, M), \forall i \in N .
$$

Our result is based on the algorithm by Procaccia and Wang [2014], which also guarantees to each agent a 2/3-approximation. However, their algorithm runs in polynomial time only for a constant number of agents. Here, we identify the source of exponentiality and take a different approach regarding certain parts of the algorithm. For 
the sake of completeness, we first present the necessary related results of Procaccia and Wang [2014], before we discuss the steps that are needed to obtain our result.

First of all, we note that even the computation of the maximin share values is already a hard problem. For a single agent $i$, the problem of deciding whether $\boldsymbol{\mu}_{i}(n, M) \geq k$ for a given $k$ is NP-complete. However, a PTAS follows by the work of Woeginger [1997]. In the original paper, which is in the context of job scheduling, Woeginger gave a PTAS for maximizing the minimum completion time on identical machines. But this scheduling problem is identical to computing a maximin partition with respect to a given agent $i$. Indeed, from agent $i$ 's perspective, it is enough to think of the machines as identical agents (the only input that we need for computing $\boldsymbol{\mu}_{i}(n, M)$ is the valuation function of $i$ ). Hence:

THEOREM 4.2 (Follows by [Woeginger 1997]). Suppose we have a set $M$ of goods to be divided among $n$ agents. Then, for each agent $i$, there exists a PTAS for approximating $\boldsymbol{\mu}_{i}(n, M)$.

A central quantity in the algorithm of Procaccia and Wang [2014] is the n-density balance parameter, denoted by $\rho_{n}$ and defined below. Before stating the definition, we give for clarity the high level idea, which can be seen as an attempt to generalize the monotonicity property of Lemma 3.4. Assume that in the course of an algorithm, we have used a subset of the items to "satisfy" some of the agents, and that those items do not have "too much" value for the rest of the agents. If $k$ is the number of remaining agents, and $S$ is the remaining set of goods, then we should expect to be able to "satisfy" these $k$ agents using the items in $S$. A good approximation in this reduced instance however, would only be an approximation with respect to $\boldsymbol{\mu}_{i}(k, S)$. Hence, in order to hope for an approximation algorithm for the original instance, we would need to examine how $\boldsymbol{\mu}_{i}(k, S)$ relates to $\boldsymbol{\mu}_{i}(n, M)$. Essentially, the parameter $\rho_{n}$ is the best guarantee one can hope to achieve for the remaining agents, based only on the fact that the complement of the set left to be shared is of relatively small value. Formally:

Definition 4.3 ([Procaccia and Wang 2014]). For any number $n$ of agents, let

$$
\rho_{n}=\max \left\{\begin{array}{ll}
\lambda \mid \begin{array}{l}
\forall M, \forall \text { additive } v_{i} \in\left(\mathbb{R}^{+}\right)^{2^{M}}, \forall S \subseteq M, \forall k, \ell \text { s.t. } k+\ell=n, \\
v_{i}(M \backslash S) \leq \ell \lambda \boldsymbol{\mu}_{i}(n, M) \Rightarrow \boldsymbol{\mu}_{i}(k, S) \geq \lambda \boldsymbol{\mu}_{i}(n, M)
\end{array}
\end{array}\right\} .
$$

After a quite technical analysis, Procaccia and Wang calculate the exact value of $\rho_{n}$ in the following lemma.

LEMma 4.4 (Lemma 3.2 of [Procaccia and Wang 2014]). For any $n \geq 2$,

$$
\rho_{n}=\frac{2\lfloor n\rfloor_{\text {odd }}}{3\lfloor n\rfloor_{\text {odd }}-1}>\frac{2}{3}
$$

where $\lfloor n\rfloor_{\text {odd }}$ denotes the largest odd integer less than or equal to $n$.

We are now ready to state our algorithm, referred to as APX-MMS (Algorithm 3 below). We elaborate on the crucial differences between Algorithm 3 and the result of Procaccia and Wang [2014] after the algorithm description (namely after Lemma 4.5). At first, the algorithm computes each agent's $\left(1-\varepsilon^{\prime}\right)$-approximate maximin value using Woeginger's PTAS, where $\varepsilon^{\prime}=\frac{3 \varepsilon}{4}$. Let $\xi=\left(\xi_{1}, \ldots, \xi_{n}\right)$ be the vector of these values. Hence, $\forall i, \boldsymbol{\mu}_{i}(n, M) \geq \xi_{i} \geq\left(1-\varepsilon^{\prime}\right) \boldsymbol{\mu}_{i}(n, M)$. Then, APX-MMS makes a call to the recursive algorithm REC-MMS (Algorithm 4 ) to compute a $\left(\frac{2}{3}-\varepsilon\right)$-approximate partition. REC-MMS takes the arguments $\varepsilon^{\prime}, n=|N|, \xi, S$ (the set of items that have not been allocated yet), $K$ (the set of agents that have not received a share of items yet), and 
the valuation functions $V_{K}=\left\{v_{i} \mid i \in K\right\}$. The guarantee provided by REC-MMS is that as long as the already allocated goods are not worth too much for the currently active agents of $K$, we can satisfy them with the remaining goods. More formally, under the assumption that

$$
\forall i \in K, \quad v_{i}(M \backslash S) \leq(n-|K|) \rho_{n} \boldsymbol{\mu}_{i}(n, M),
$$

which we will show that it holds before each call, REC-MMS $\left(\varepsilon^{\prime}, n, \boldsymbol{\xi}, S, K, V_{K}\right)$ computes a $|K|$-partition of $S$, so that each agent receives items of value at least $\left(1-\varepsilon^{\prime}\right) \rho_{n} \xi_{i}$.

The initial call of the recursion is, of course, $\operatorname{REC}-\operatorname{MMS}\left(\varepsilon^{\prime}, n, \boldsymbol{\xi}, M, N, V_{N}\right)$. Before moving on to the next recursive call, REC-MMS appropriately allocates some of the items to some of the agents, so that they receive value at least $\left(1-\varepsilon^{\prime}\right) \rho_{n} \xi_{i}$ each. This is achieved by identifying an appropriate matching between some currently unsatisfied agents and certain bundles of items, as described in the algorithm. In particular, the most important step in the algorithm is to first compute the set $X^{+}$(line 6), which is the set of agents that will not be matched in the current call. The remaining active agents, i.e., $K \backslash X^{+}$, are then guaranteed to get matched in the current round, whereas $X^{+}$will be satisfied in the next recursive calls. In order to ensure this for $X^{+}$, REC-MMS guarantees that inequality (1) holds for $K=X^{+}$and with $S$ being the rest of the items. Note that (1) trivially holds for the initial call of REC-MMS, where $K=N$ and $S=M$.

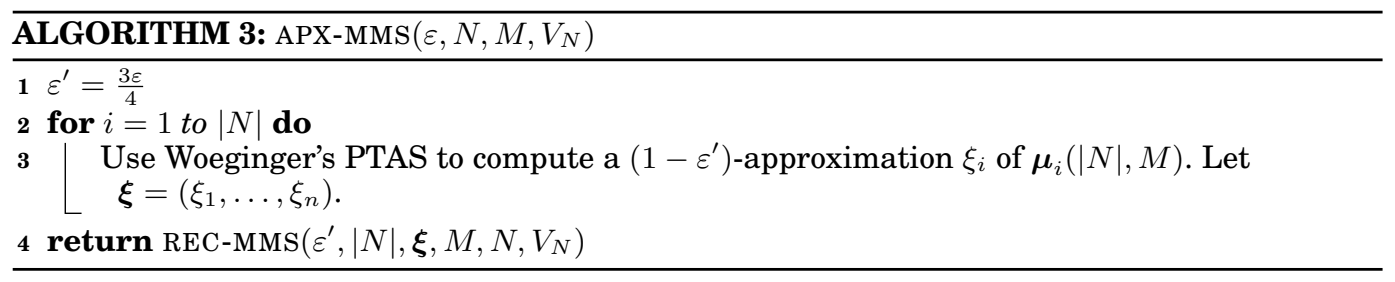

For simplicity, in the description of REC-MMS, we assume that $K=\{1,2, \ldots,|K|\}$. Also, for the bipartite graph defined below in the algorithm, by $\Gamma\left(X^{+}\right)$we denote the set of neighbors of the vertices in $X^{+}$.

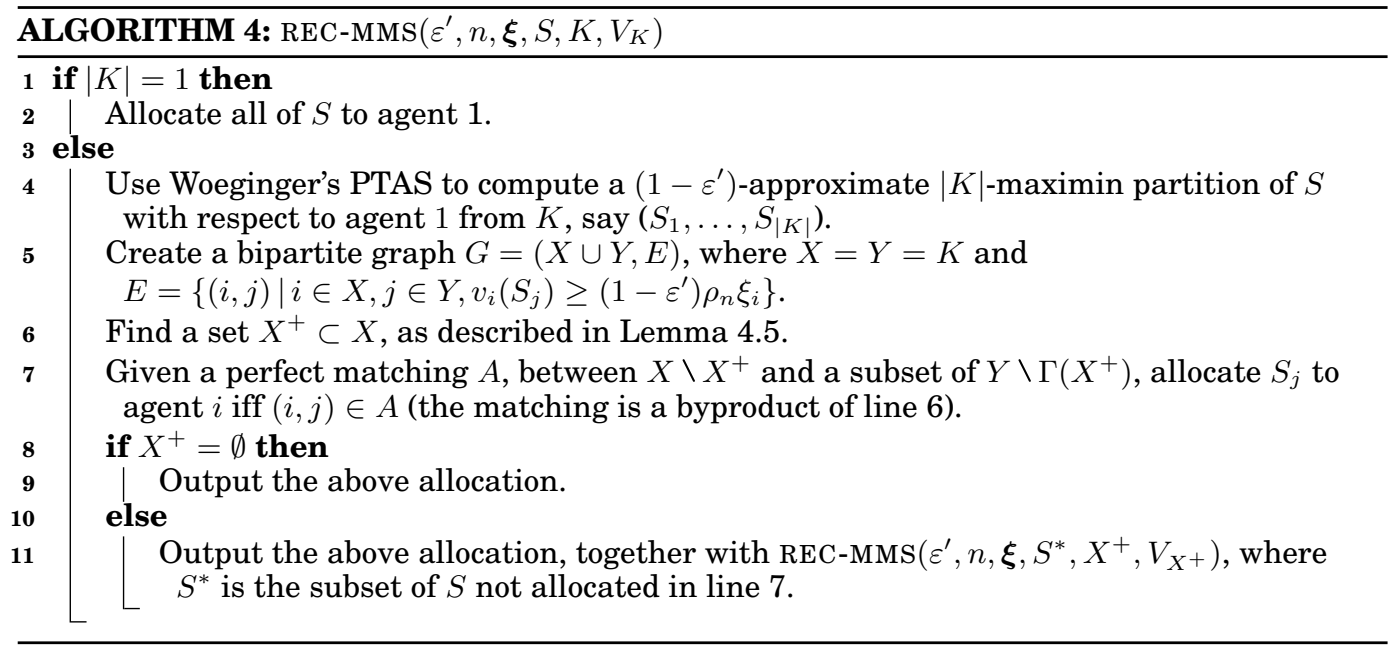

To proceed with the analysis, and since the choice of $X^{+}$plays an important role (line 6 of Algorithm 4), we should first clarify what properties of $X^{+}$are needed for the algorithm to work. The following lemma is the most crucial part in the design of our algorithm. 
LEMMA 4.5. Assume that for $n, M, S, K, V_{K}$ inequality (1) holds and let $G=(X \cup$ $Y, E)$ be the bipartite graph defined in line 5 of REC-MMS. Then there exists a subset $X^{+}$ of $X \backslash\{1\}$, such that:

(i) $X^{+}$can be found efficiently.

(ii) There exists a perfect matching between $X \backslash X^{+}$and a subset of $Y \backslash \Gamma\left(X^{+}\right)$.

(iii) If we allocate subsets to agents according to such a matching (as described in line 7) and $X^{+} \neq \emptyset$, then inequality (1) holds for $n, M, S^{*}, X^{+}, V_{X^{+}}$where $S^{*} \subseteq S$ is the unallocated set of items, i.e.:

$$
\forall i \in X^{+}, \quad v_{i}\left(M \backslash S^{*}\right) \leq\left(n-\left|X^{+}\right|\right) \rho_{n} \boldsymbol{\mu}_{i}(n, M) .
$$

Before we prove Lemma 4.5, we elaborate on the main differences between our setup and the approach of Procaccia and Wang [2014]:

Choice of $X^{+}$. In Procaccia and Wang [2014], $X^{+}$is defined as $\arg \max _{Z \subseteq K \backslash\{1\}}\{|Z||| Z|\geq| \Gamma(Z) \mid\}$. Clearly, when $n$ is constant, so is $|K|$, and thus the computation of $X^{+}$is trivial. However, it is not clear how to efficiently find such a set in general, when $n$ is not constant. We propose a definition of $X^{+}$, which is efficiently computable and has the desired properties. In short, our $X^{+}$is any appropriately selected counterexample to Hall's Theorem for the graph $G$ constructed in line 5.

Choice of $\varepsilon$. The algorithm works for any $\varepsilon>0$, but Procaccia and Wang [2014] choose an $\varepsilon$ that depends on $n$, and it is such that $(1-\varepsilon) \rho_{n} \geq \frac{2}{3}$. This is possible since for any $n, \rho_{n} \geq \frac{2}{3}\left(1+\frac{1}{3 n-1}\right)$. However, in this case, the running time of Woeginger's PTAS (line 4 ) is not polynomial in $n$. Here, we consider any fixed $\varepsilon$, independent of $n$, hence the approximation ratio of $\frac{2}{3}-\varepsilon$.

The formal definition of $X^{+}$is given within the proof of Lemma 4.5 that follows.

Proof of Lemma 4.5. We will show that either $X^{+}=\emptyset$ (in the case where $G$ has a perfect matching), or some set $X^{+}$with $X^{+} \in\{Z \subseteq X:|Z|>|\Gamma(Z)| \wedge \exists$ matching of size $|X \backslash Z|$ in $G \backslash\{Z \cup \Gamma(Z)\}\}$ has the desired properties. Moreover, we propose a way to find such a set efficiently. We first find a maximum matching $B$ of $G$. If $|B|=|K|$, then we are done, since for $X^{+}=\emptyset$, properties (i) and (ii) of Lemma 4.5 hold, while we need not check (iii). If $|B|<|K|$, then there must be a subset of $X$ violating the condition of Hall's Theorem. ${ }^{1}$ Let $X_{u}, X_{m}$ be the partition of $X$ in unmatched and matched vertices respectively, according to $B$, with $X_{u} \neq \emptyset, X_{m} \neq \emptyset$. Similarly, we define $Y_{u}, Y_{m}$.

We now construct a directed graph $G^{\prime}=\left(X \cup Y, E^{\prime}\right)$, where we direct all edges of $G$ from $X$ to $Y$, and on top of that, we add one copy of each edge of the matching but with direction from $Y$ to $X$. In particular, $\forall i \in X, \forall j \in Y$, if $(i, j) \in E$ then $(i, j) \in E^{\prime}$, and moreover if $(i, j) \in B$ then $(j, i) \in E^{\prime}$. We claim that the following set satisfies the desired properties

$$
X^{+}:=X_{u} \cup\left\{v \in X: v \text { is reachable from } X_{u} \text { in } G^{\prime}\right\} .
$$

Note that $X^{+}$is easy to compute; after finding the maximum matching in $G$, and constructing $G^{\prime}$, we can run a depth-first search in each connected component of $G^{\prime}$, starting from the vertices of $X_{u}$. See also Figure 1, after the proof of Theorem 4.1 for an illustration.

\footnotetext{
${ }^{1}$ The special case of Hall's Theorem [Hall 1935] used here, states that given a bipartite graph $G=(X \cup Y, E)$, where $X, Y$ are disjoint independent sets with $|X|=|Y|$, there is a perfect matching in $G$ if and only if $|W| \leq|\Gamma(W)|$ for every $W \subseteq X$.
} 
Given the definition of $X^{+}$, we now show property (ii). Back to the original graph $G$, we first claim that $\left|X^{+}\right|>\left|\Gamma\left(X^{+}\right)\right|$. To prove this, note that if $j \in \Gamma\left(X^{+}\right)$in $G$, then $j \in Y_{m}$. If not, then it is not difficult to see that there is an augmenting path from a vertex in $X_{u}$ to $j$, which contradicts the maximality of $B$. Indeed, since $j \in \Gamma\left(X^{+}\right)$, let $i$ be a neighbor of $j$ in $X^{+}$. If $i \in X_{u}$, then the edge $(i, j)$ would enlarge the matching. Otherwise, $i \in X_{m}$ and since also $i \in X^{+}$, there is a path in $G^{\prime}$ from some vertex of $X_{u}$ to $i$. But this path by construction of the directed graph $G^{\prime}$ must consist of an alternation of unmatched and matched edges, hence together with $(i, j)$ we have an augmenting path.

Therefore, $\Gamma\left(X^{+}\right) \subseteq Y_{m}$, i.e., for any $j \in \Gamma\left(X^{+}\right)$, there is an edge $(i, j)$ in the matching $B$. But then $i$ has to belong to $X^{+}$by the construction of $G^{\prime}$ (and since $j \in \Gamma\left(X^{+}\right)$). To sum up: for any $j \in \Gamma\left(X^{+}\right)$, there is exactly one distinct vertex $i$, with $(i, j) \in E$, and $i \in X^{+} \cap X_{m}$, i.e., $\left|X^{+} \cap X_{m}\right| \geq\left|\Gamma\left(X^{+}\right)\right|$. In fact, we have equality here, because it is also true that for any $i \in X^{+} \cap X_{m}$, there is a distinct vertex $j \in Y_{m}$ which is trivially reachable from $X^{+}$. Hence, $\left|X^{+} \cap X_{m}\right|=\left|\Gamma\left(X^{+}\right)\right|$. Since $X_{u} \neq \emptyset$, we have $\left|X^{+}\right|=\left|X_{u}\right|+\left|X^{+} \cap X_{m}\right| \geq 1+\left|\Gamma\left(X^{+}\right)\right|$. So, $\left|X^{+}\right|>\left|\Gamma\left(X^{+}\right)\right|$.

Also, note that $X^{+} \subseteq X \backslash\{1\}$, because for any $Z \subseteq X$ that contains vertex 1 we have $|\Gamma(Z)|=|K| \geq|Z|$. This is due to the fact that for any vertex $j \in Y$, the edge $(1, j)$ is present by the construction, since $v_{1}\left(S_{j}\right) \geq\left(1-\varepsilon^{\prime}\right) \boldsymbol{\mu}_{1}(k, S) \geq\left(1-\varepsilon^{\prime}\right) \rho_{n} \boldsymbol{\mu}_{1}(n, M) \geq$ $\left(1-\varepsilon^{\prime}\right) \rho_{n} \xi_{1}$, for all $1 \leq j \leq|K|$.

We now claim that if we remove $X^{+}$and $\Gamma\left(X^{+}\right)$from $G$, then the restriction of $B$ on the remaining graph, still matches all vertices of $X \backslash X^{+}$, establishing property (ii). Indeed, note first that for any $i \in X \backslash X^{+}$, it has to hold that $i \in X_{m}$, since $X^{+}$ contains $X_{u}$. Also, for any edge $(i, j) \in B$ with $i \in X$ and $j \in \Gamma\left(X^{+}\right)$, we have $i \in X^{+}$ by the construction of $X^{+}$. So, for any $i \in X \backslash X^{+}$, its pair in $B$ belongs to $Y \backslash \Gamma\left(X^{+}\right)$. Equivalently, $B$ induces a perfect matching between $X \backslash X^{+}$and a subset of $Y \backslash \Gamma\left(X^{+}\right)$ (this is the matching $A$ in line 7 of the algorithm).

What is left to prove is that property (iii) also holds for $X^{+}$. This can be done by the same arguments as in Procaccia and Wang [2014], specifically by the following lemma which can be inferred from their work.

LEMma 4.6 ([Procaccia and Wang 2014], end of Subsection 3.1). Assume that inequality (1) holds for $n, M, S, K, V_{K}$, and let $G$ be the graph defined in line 5. For any $Z \subseteq X$, if there exists a perfect matching between $X \backslash Z$ and a subset of $Y \backslash \Gamma(Z)$, say $Y^{*}$, and there are no edges between $Z$ and $Y^{*}$ in $G$, then property (iii) holds as well.

Clearly, there are no edges between $X^{+}$and $Y \backslash \Gamma\left(X^{+}\right)$. Hence, Lemma 4.6 can be applied to $X^{+}$, completing the proof.

Given Lemma 4.5, we can now prove the main result of this section, the correctness of APX-MMS.

Proof OF THEOREM 4.1. It is clear that the running time of the algorithm is polynomial. Its correctness is based on the correctness of REC-MMS. The latter can be proven with strong induction on $|K|$, the number of still active agents that REC-MMS receives as input, under the assumption that (1) holds before each new call of REC-MMS (which we have established by Lemma 4.5). For $|K|=1$, assuming that inequality (1) holds, we have for agent 1 of $K$ :

$$
\begin{aligned}
v_{1}(S) & =v_{1}(M)-v_{1}(M \backslash S) \geq n \boldsymbol{\mu}_{1}(n, M)-(n-1) \rho_{n} \boldsymbol{\mu}_{1}(n, M) \\
& \geq \boldsymbol{\mu}_{1}(n, M) \geq\left(\frac{2}{3}-\varepsilon\right) \boldsymbol{\mu}_{1}(n, M) .
\end{aligned}
$$


For the inductive step, Lemma 4.5 and the choice of $X^{+}$are crucial. Consider an execution of REC-MMS during which some agents will receive a subset of items and the rest will form the set $X^{+}$to be handled recursively. For all the agents in $X^{+}$-if anywe are guaranteed $\left(\frac{2}{3}-\varepsilon\right)$-approximate shares by property (iii) of Lemma 4.5 and by the inductive hypothesis. On the other hand, for each agent $i$ that receives a subset $S_{j}$ of items in line 7 , we have

$$
v_{i}\left(S_{j}\right) \geq\left(1-\varepsilon^{\prime}\right) \rho_{n} \xi_{i} \geq\left(1-\varepsilon^{\prime}\right)^{2} \rho_{n} \boldsymbol{\mu}_{i}(n, M)>\left(1-2 \varepsilon^{\prime}\right) \frac{2}{3} \boldsymbol{\mu}_{i}(n, M)=\left(\frac{2}{3}-\varepsilon\right) \boldsymbol{\mu}_{i}(n, M),
$$

where the first inequality holds because $(i, j) \in E(G)$.

In Figure 1, we give a simple snapshot to illustrate a recursive call of REC-MMS. In particular, in Subfigure 1(a), we see a bipartite graph $G$ that could be the current configuration for REC-MMS, along with a maximum matching. In Subfigure 1(b), we see the construction of $G^{\prime}$, as described in Lemma 4.5, and the set $X^{+}$. The bold (black) edges in $G^{\prime}$ signify that both directions are present. The set $X^{+}$consists then of $X_{u}$ and all other vertices of $X$ reachable from $X_{u}$. Finally, Subfigure 1(b) also shows the set of agents that are satisfied in the current call along with the corresponding perfect matching, as claimed in Lemma 4.5.

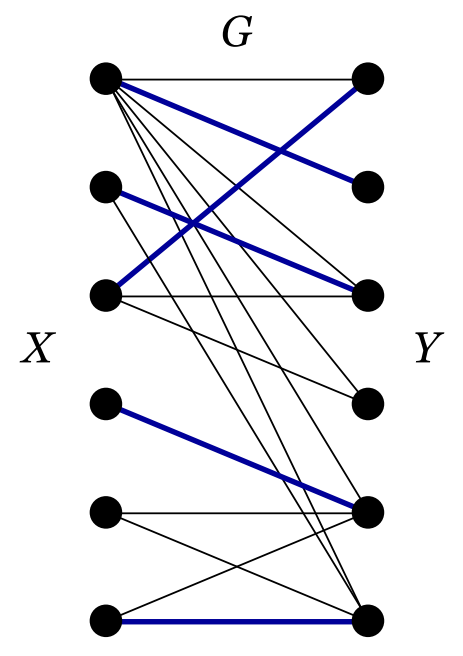

(a) The graph $G$ defined in line 5 of Algorithm 4 shown with a maximum matching (blue edges). Agent 1 is the top vertex of $X$.

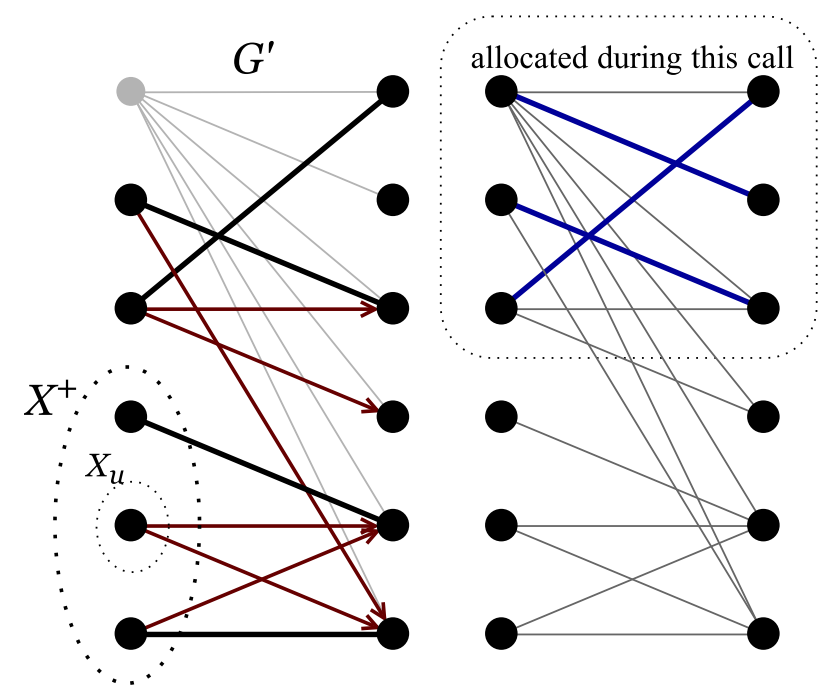

(b) The graph $G^{\prime}$ defined in the proof of Lemma 4.5, where for clarity, agent 1 and her edges are grayed out. The black edges signify that both directions are present, i.e., they correspond to pairs of anti-parallel edges. On the right we show the actual allocation resulting from $G$.

Fig. 1. Ilustration of $G, G^{\prime}$ and $X^{+}$.

We note that the analysis of the algorithm is tight, given the analysis on $\rho_{n}$ (see Section 3.3 of Procaccia and Wang [2014]). Improving further on the approximation ratio of $2 / 3$ seems to require drastically new ideas and it is a challenging open problem. We stress that even a PTAS is not currently ruled out by the lower bound constructions [Kurokawa et al. 2016; Procaccia and Wang 2014]. Related to this, in the next section we consider two special cases in which we can obtain better positive results. 


\section{TWO SPECIAL CASES}

In this section, we consider two interesting special cases, where we have improved approximations. The first is the case of $n=3$ agents, where we obtain a $7 / 8$ approximation, improving on the 3/4-approximation of Procaccia and Wang [2014]. The second is the case where all values for the goods belong to $\{0,1,2\}$. This is an extension of the $\{0,1\}$ setting discussed in Bouveret and Lemaitre [2016], and we show how to get an exact allocation without any approximation loss.

\subsection{The Case of $n=3$ Agents}

For $n=2$, it is pointed out in Bouveret and Lemaître [2016] that maximin share allocations exist via an analog of the cut and choose protocol. Using the PTAS of Woeginger [1997], we can then have a $(1-\varepsilon)$-approximation in polynomial time. In contrast, as soon as we move to $n=3$, things become more interesting. It is proven that with 3 agents there exist instances where no maximin share allocation exists [Procaccia and Wang 2014]. The best known approximation guarantee is $\frac{3}{4}$ by observing that the quantity $\rho_{n}$, defined in Section 4 , satisfies $\rho_{3} \geq \frac{3}{4}$.

We provide a different algorithm, improving the approximation to $\frac{7}{8}-\varepsilon$. To do this, we combine ideas from both algorithms presented so far in Sections 3 and 4 . The main result of this subsection is as follows:

THEOREM 5.1. Let $N=\{1,2,3\}$ be a set of three agents with additive valuations, and let $M$ be a set of goods. For any constant $\varepsilon>0$, Algorithm 5 produces in polynomial time an allocation $\left(S_{1}, S_{2}, S_{3}\right)$, such that

$$
v_{i}\left(S_{i}\right) \geq\left(\frac{7}{8}-\varepsilon\right) \boldsymbol{\mu}_{i}(3, M), \forall i \in N .
$$

The algorithm is shown below. Before we prove Theorem 5.1, we provide here a brief outline of how the algorithm works.

Algorithm Outline: First, approximate values for the $\mu_{i} \mathrm{~s}$ are calculated as before. Then, if there are items with large value to some agent, in analogy to Algorithm 2, we first allocate one of those reducing this way the problem to the simple case of $n=2$. If there are no items of large value, then the first agent partitions the items as in Algorithm 4. In the case where this partition does not satisfy all three agents, then the second agent repartitions two of the bundles of the first agent. Actually, she tries two different such repartitions, and we show that at least one of them works out. The definition of a bipartite preference graph and a corresponding matching (as in Algorithm 4) is never mentioned explicitly here. However, the main idea (and the difference with Algorithm 4) is that if there are several ways to pick a perfect matching between $X \backslash X^{+}$and a subset of $Y \backslash \Gamma\left(X^{+}\right)$, then we try them all and choose the best one. Of course, since $n=3$, if there is no perfect matching in the preference graph, then $X \backslash X^{+}$ is going to be just a single vertex, and we only have to examine two possible perfect matchings between $X \backslash X^{+}$and a subset of $Y \backslash \Gamma\left(X^{+}\right)$.

Proof of TheOREM 5.1. First, note that for constant $\varepsilon$ the algorithm runs in time polynomial in $|M|$. Next, we prove the correctness of the algorithm.

If the output is computed in lines 3-4 then for agent $i$, as defined in line 3 , the value she receives is at least $\frac{7}{8} \xi_{i} \geq \frac{7}{8}(1-\varepsilon) \boldsymbol{\mu}_{i}(3, M)>\left(\frac{7}{8}-\varepsilon\right) \boldsymbol{\mu}_{i}(3, M)$. The remaining two agents $i_{1}, i_{2}$ essentially apply an approximate version of a cut and choose protocol. Agent $i_{1}$ computes a $(1-\varepsilon)$-approximate 2-maximin partition of $M \backslash\{j\}$, say $\left(C_{1}, C_{2}\right)$, then agent $i_{2}$ takes the set she prefers among $C_{1}$ and $C_{2}$, and agent $i_{1}$ gets the other. By the monotonicity lemma (Lemma 3.4), we know that $\boldsymbol{\mu}_{i_{1}}(2, M \backslash\{j\}) \geq \boldsymbol{\mu}_{i_{1}}(3, M)$, 


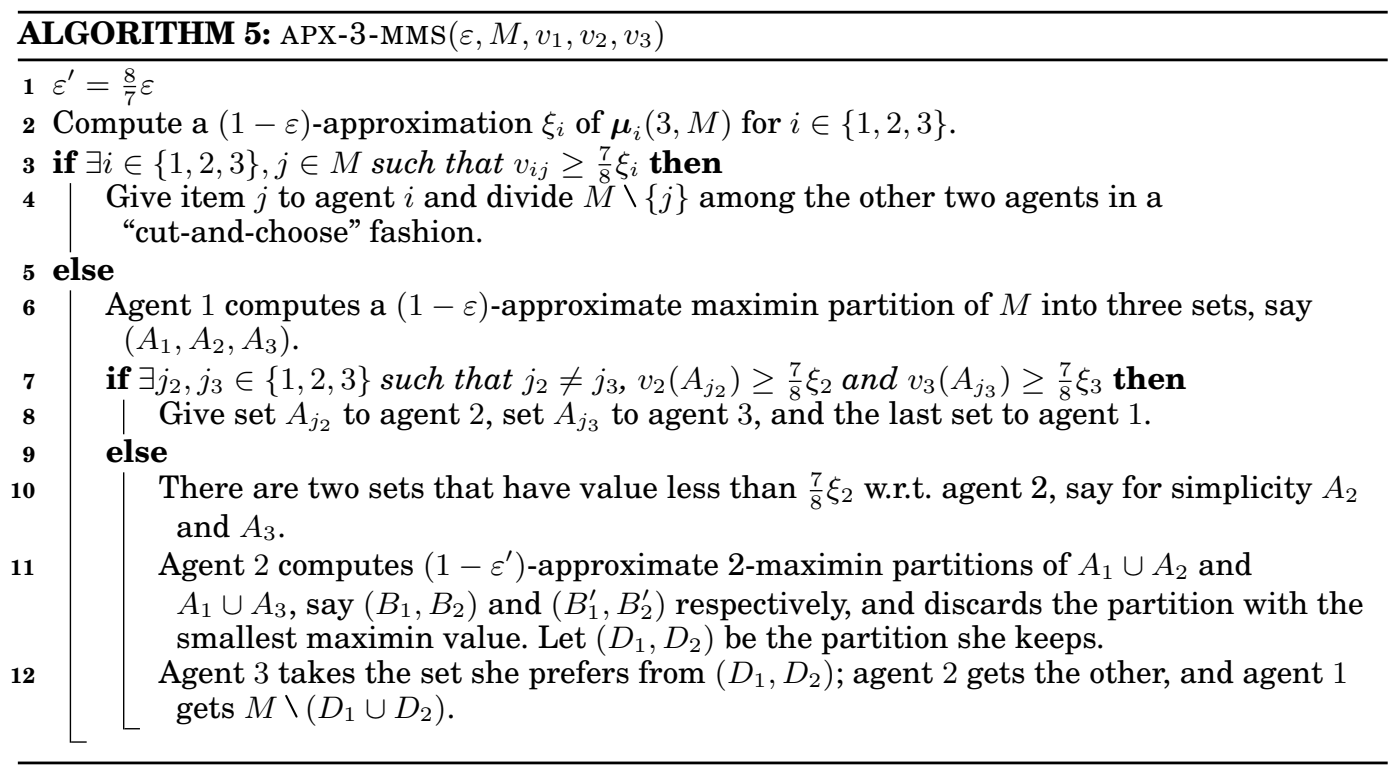

and thus no matter which set is left for agent $i_{1}$, she is guaranteed a total value of at least $(1-\varepsilon) \boldsymbol{\mu}_{i_{1}}(3, M)>\left(\frac{7}{8}-\varepsilon\right) \boldsymbol{\mu}_{i_{1}}(3, M)$. Similarly, we have $\boldsymbol{\mu}_{i_{2}}(2, M \backslash\{j\}) \geq \boldsymbol{\mu}_{i_{2}}(3, M)$, and therefore $v_{i_{2}}(M \backslash\{j\}) \geq 2 \boldsymbol{\mu}_{i_{2}}(3, M)$. Since $i_{2}$ chooses before $i_{1}$, she is guaranteed a total value that is at least $\boldsymbol{\mu}_{i_{2}}(3, M)>\left(\frac{7}{8}-\varepsilon\right) \boldsymbol{\mu}_{i_{2}}(3, M)$.

If the output is computed in lines 6-8 then clearly all agents receive a $(7 / 8-\varepsilon)$ approximation, since for agent 1 it does not matter which of the $A_{i} \mathrm{~s}$ she gets.

The most challenging case is when the output is computed in lines 10-12 (starting with the partition from line 6). Then, as before, agent 1 receives a value that is at least a $(7 / 8-\varepsilon)$-approximation no matter which of the three sets she gets. For agents 2 and 3 , however, the analysis is not straightforward. We need the following lemma.

LEMMA 5.2. Let $N, M, \varepsilon$ be as above, such that for all $j \in M$ we have $v_{2 j}<\frac{7}{8} \xi_{2}$. Consider any partition of $M$ into 3 sets $A_{1}, A_{2}, A_{3}$ and assume that there are no $j_{2}, j_{3} \in\{1,2,3\}$ such that $j_{2} \neq j_{3}, v_{2}\left(A_{j_{2}}\right) \geq \frac{7}{8} \xi_{2}$ and $v_{3}\left(A_{j_{3}}\right) \geq \frac{7}{8} \xi_{3}$. Then lines 10-12 of Algorithm 5 produce an allocation $\left(S_{2}, S_{3}\right)$ for agents 2 and 3 , such that for $i \in\{2,3\}$ : $v_{i}\left(S_{i}\right) \geq\left(\frac{7}{8}-\varepsilon\right) \boldsymbol{\mu}_{i}(3, M)$. Moreover, if agent 1 is given set $A_{k}$, then $S_{2} \cup S_{3}=\bigcup_{\ell \in N \backslash k} A_{\ell}$.

Clearly, Lemma 5.2 completes the proof.

Before stating the proof of Lemma 5.2, we should mention how it is possible to go beyond the previously known $\frac{3}{4}$-approximation. As noted above, $\rho_{n}$ is by definition the best guarantee we can get, based only on the fact that the complement of the set left to be shared is not too large. As a result, the $\frac{7}{8}$ ratio cannot be guaranteed just by the excess value. Instead, in addition to making sure that the remaining items are valuable enough for the remaining agents, we further argue about how a maximin partition would distribute those items.

There is an alternative interpretation of Algorithm 5 in terms of Algorithm 3. Whenever only a single agent (i.e., agent 1 ) is going to become satisfied in the first recursive call, we try all possible maximum matchings of the graph $G$ for the calculation of $X^{+}$. Then we proceed with the "best" such matching. Here, for $n=3$, this means we only 
have to consider two possibilities for the set agent 1 is going to get matched to; it is either $A_{2}$ or $A_{3}$ (subject to the assumptions in Algorithm 5).

Proof of Lemma 5.2. First, recall that $v_{2}(M) \geq 3 \boldsymbol{\mu}_{2}(3, M) \geq 3 \xi_{2}$. Like in the description of the algorithm we may assume that agent 1 gets set $A_{3}$, without loss of generality. Before we move to the analysis we should lay down some facts. Let $\left(B_{1}, B_{2}\right)$ be agent 2's $\left(1-\varepsilon^{\prime}\right)$-approximate maximin partition of $A_{1} \cup A_{2}$ computed in line 11; similarly $\left(B_{1}^{\prime}, B_{2}^{\prime}\right)$ is agent 2's $\left(1-\varepsilon^{\prime}\right)$-approximate maximin partition of $A_{1} \cup A_{3}$. We may assume that $v_{2}\left(B_{1}\right) \geq v_{2}\left(B_{2}\right)$. Also, assume that in line 11 of the algorithm we have $\left(D_{1}, D_{2}\right)=\left(B_{1}, B_{2}\right)$, i.e., $\min \left\{v_{2}\left(B_{1}^{\prime}\right), v_{2}\left(B_{2}^{\prime}\right)\right\} \leq v_{2}\left(B_{2}\right)$ and $M \backslash\left(D_{1} \cup D_{2}\right)=A_{3}$. The case where $\left(D_{1}, D_{2}\right)=\left(B_{1}^{\prime}, B_{2}^{\prime}\right)$ is symmetric. Our goal is to show that $v_{2}\left(B_{2}\right) \geq\left(\frac{7}{8}-\varepsilon\right) \boldsymbol{\mu}_{2}(3, M)$. For simplicity, we write $\boldsymbol{\mu}_{2}$ instead of $\boldsymbol{\mu}_{2}(3, M)$.

Note, towards a contradiction, that

$$
\begin{aligned}
v_{2}\left(B_{2}\right) & <\left(\frac{7}{8}-\varepsilon\right) \boldsymbol{\mu}_{2} \Rightarrow \\
\left(1-\varepsilon^{\prime}\right) \boldsymbol{\mu}_{2}\left(2, A_{1} \cup A_{2}\right) & <\left(\frac{7}{8}-\varepsilon\right) \boldsymbol{\mu}_{2} \Rightarrow \\
\left(1-\varepsilon^{\prime}\right) \boldsymbol{\mu}_{2}\left(2, A_{1} \cup A_{2}\right) & <\left(\frac{7}{8}-\frac{7}{8} \varepsilon^{\prime}\right) \boldsymbol{\mu}_{2} \Rightarrow \\
\boldsymbol{\mu}_{2}\left(2, A_{1} \cup A_{2}\right) & <\frac{7}{8} \boldsymbol{\mu}_{2} .
\end{aligned}
$$

Moreover, this means $\min \left\{v_{2}\left(B_{1}^{\prime}\right), v_{2}\left(B_{2}^{\prime}\right)\right\}<\left(\frac{7}{8}-\varepsilon\right) \boldsymbol{\mu}_{2}$ as well, which leads to $\boldsymbol{\mu}_{2}\left(2, A_{1} \cup A_{3}\right)<\frac{7}{8} \boldsymbol{\mu}_{2}$. So, it suffices to show that either $\boldsymbol{\mu}_{2}\left(2, A_{1} \cup A_{2}\right)$ or $\boldsymbol{\mu}_{2}\left(2, A_{1} \cup A_{3}\right)$ is at least $\frac{7}{8} \boldsymbol{\mu}_{2}$. This statement is independent of the $B_{i}$ s and in what follows we consider exact maximin partitions with respect to agent 2 . Before we proceed, we should make clear that for the case we are analyzing there are indeed exactly two sets in $\left\{A_{1}, A_{2}, A_{3}\right\}$ each with value less than $\frac{7}{8} \boldsymbol{\mu}_{2}$ with respect to agent 2 , as claimed in line 10 of the algorithm. Indeed, notice that in any partition of $M$ there is at least one set with value at least $\boldsymbol{\mu}_{2}$ with respect to agent 2 , due to the fact that $v_{2}(M) \geq 3 \boldsymbol{\mu}_{2}$ and by the definition of a maximin partition. If, however, there were at least 2 sets in $\left\{A_{1}, A_{2}, A_{3}\right\}$ with value at least $\frac{7}{8} \xi_{2}$, then we would be at the case handled in steps 6-8. Hence, there will be exactly two sets each with value less than $\frac{7}{8} \xi_{2} \leq \frac{7}{8} \boldsymbol{\mu}_{2}$ for agent 2 and as stated in the algorithm we assume these are the sets $A_{2}, A_{3}$.

Consider a 3-maximin share allocation $\left(A_{1}^{\prime}, A_{2}^{\prime}, A_{3}^{\prime}\right)$ of $M$ with respect to agent 2 . Let $F_{i}=A_{i}^{\prime} \cap A_{3}$ for $i=1,2,3$. Without loss of generality, we may assume that $v_{2}\left(F_{1}\right) \leq$ $v_{2}\left(F_{2}\right) \leq v_{2}\left(F_{3}\right)$.

If $v_{2}\left(F_{1}\right) \leq \frac{1}{8} \boldsymbol{\mu}_{2}$, then the partition $\left(A_{1}^{\prime} \backslash A_{3},\left(A_{2}^{\prime} \cup A_{3}^{\prime}\right) \backslash A_{3}\right)$ is a partition of $A_{1} \cup A_{2}$ such that

$$
v_{2}\left(A_{1}^{\prime} \backslash A_{3}\right)=v_{2}\left(A_{1}^{\prime}\right)-v_{2}\left(F_{1}\right) \geq \boldsymbol{\mu}_{2}-\frac{1}{8} \boldsymbol{\mu}_{2}=\frac{7}{8} \boldsymbol{\mu}_{2}
$$

and

$$
v_{2}\left(\left(A_{2}^{\prime} \cup A_{3}^{\prime}\right) \backslash A_{3}\right) \geq v_{2}\left(A_{2}^{\prime}\right)+v_{2}\left(A_{3}^{\prime}\right)-v_{2}\left(A_{3}\right) \geq 2 \boldsymbol{\mu}_{2}-\frac{7}{8} \boldsymbol{\mu}_{2}=\frac{9}{8} \boldsymbol{\mu}_{2} .
$$

So, in this case we conclude that $\boldsymbol{\mu}_{2}\left(2, A_{1} \cup A_{2}\right) \geq \frac{7}{8} \boldsymbol{\mu}_{2}$.

On the other hand, if $v_{2}\left(F_{1}\right)>\frac{1}{8} \boldsymbol{\mu}_{2}$ we are going to show that $\boldsymbol{\mu}_{2}\left(2, A_{1} \cup A_{3}\right) \geq \frac{7}{8} \boldsymbol{\mu}_{2}$. Towards this we consider a 2-maximin share allocation $\left(C_{1}, C_{2}\right)$ of $A_{1}$ with respect to agent 2 and let us assume that $v_{2}\left(C_{1}\right) \geq v_{2}\left(C_{2}\right)$. For a rough depiction of the different sets involved in the following arguments, see Figure 2. 

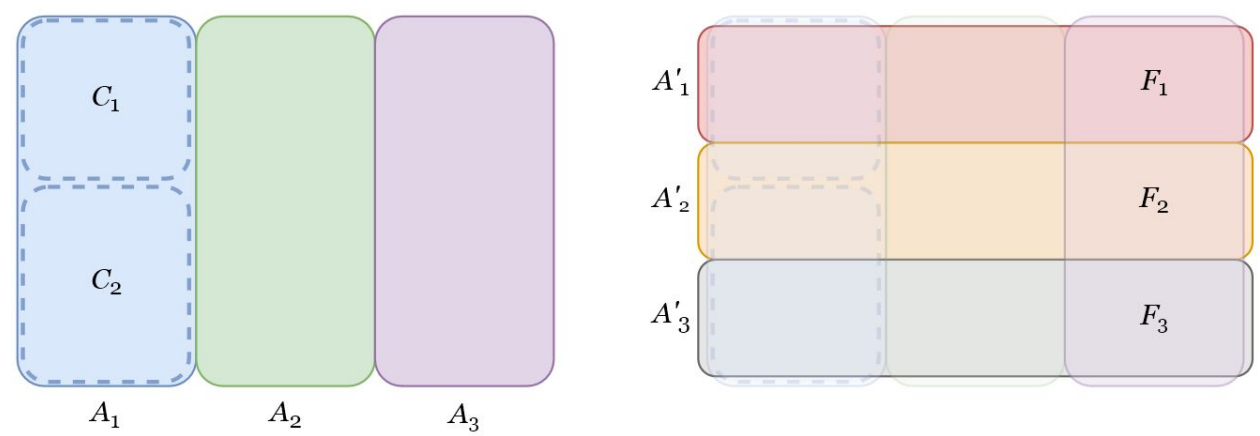

Fig. 2. Assuming that the set of items $M$ is represented by a rectangle, here is a depiction of several sets involved in the proof of Lemma 5.2. Recall that $\left(A_{1}, A_{2}, A_{3}\right)$ and $\left(A_{1}^{\prime}, A_{2}^{\prime}, A_{3}^{\prime}\right)$ are partitions of $M,\left(C_{1}, C_{2}\right)$ is a partition of $A_{1}$, and $F_{i}=A_{i}^{\prime} \cap A_{3}$ for $i=1,2,3$.

Claim 5.3. For $C_{1}, C_{2}, A_{3}, F_{1}, F_{2}, F_{3}$ as above, we have

(i) $v_{2}\left(A_{3}\right)+v_{2}\left(C_{2}\right) \geq \frac{7}{8} \boldsymbol{\mu}_{2}$, and

(ii) $v_{2}\left(F_{1}\right)+v_{2}\left(F_{2}\right)+v_{2}\left(C_{1}\right)>\frac{7}{8} \boldsymbol{\mu}_{2}$.

PRoof. Note that

$$
v_{2}\left(C_{1}\right)+v_{2}\left(C_{2}\right)+v_{2}\left(A_{3}\right)=v_{2}(M)-v_{2}\left(A_{2}\right)>3 \boldsymbol{\mu}_{2}-\frac{7}{8} \boldsymbol{\mu}_{2}=\frac{17}{8} \boldsymbol{\mu}_{2} .
$$

If $v_{2}\left(A_{3}\right)+v_{2}\left(C_{2}\right)<\frac{7}{8} \boldsymbol{\mu}_{2}$ then $v_{2}\left(C_{1}\right)>\frac{10}{8} \boldsymbol{\mu}_{2}$. Moreover,

$$
v_{2}\left(A_{3}\right)=v_{2}\left(F_{1}\right)+v_{2}\left(F_{2}\right)+v_{2}\left(F_{3}\right) \geq 3 v_{2}\left(F_{1}\right)>\frac{3}{8} \boldsymbol{\mu}_{2},
$$

so $v_{2}\left(A_{3}\right)+v_{2}\left(C_{2}\right)<\frac{7}{8} \boldsymbol{\mu}_{2}$ implies that $v_{2}\left(C_{2}\right)<\frac{4}{8} \boldsymbol{\mu}_{2}$.

Let $d$ denote the difference $v_{2}\left(C_{1}\right)-v_{2}\left(C_{2}\right)$; clearly $d>\frac{6}{8} \boldsymbol{\mu}_{2}$. It is not hard to see that $\min _{j \in C_{1}} v_{2 j} \geq d$. Indeed, suppose there existed some $j \in C_{1}$ such that $v_{2 j}<d$. Then, by moving $j$ from $C_{1}$ to $C_{2}$ we increase the minimum value of the partition, which contradicts the choice of $\left(C_{1}, C_{2}\right)$.

Since $v_{2}\left(C_{1}\right)>\frac{10}{8} \boldsymbol{\mu}_{2}$ and no item has value more than $\frac{7}{8} \boldsymbol{\mu}_{2}$ for agent 2 , this means that $C_{1}$ contains at least two items. Thus, $v_{2}\left(C_{1}\right) \geq \min _{j \in C_{1}} v_{2 j}>\frac{12}{8} \boldsymbol{\mu}_{2}$.

Now, for any item $g \in \arg \min _{j \in C_{1}} v_{2 j}$, the partition $\left(\{g\}, A_{1} \backslash\{g\}\right)$ is strictly better than $\left(C_{1}, C_{2}\right)$, since $v_{2 g}>\frac{6}{8} \boldsymbol{\mu}_{2}>v_{2}\left(C_{2}\right)$ and $v_{2}\left(A_{1} \backslash\{g\}\right)=v_{2}\left(A_{1}\right)-v_{2 g} \geq v_{2}\left(C_{1}\right)-v_{2 g}>$ $\frac{12}{8} \boldsymbol{\mu}_{2}-\frac{6}{8} \boldsymbol{\mu}_{2}=\frac{6}{8} \boldsymbol{\mu}_{2}>v_{2}\left(C_{2}\right)$. Again, this contradicts the choice of $\left(C_{1}, C_{2}\right)$. Hence, it must be that $v_{2}\left(A_{3}\right)+v_{2}\left(C_{2}\right) \geq \frac{7}{8} \boldsymbol{\mu}_{2}$.

The proof of (ii) is simpler. Notice that

$$
\begin{aligned}
v_{2}\left(F_{1}\right)+v_{2}\left(F_{2}\right)+v_{2}\left(C_{1}\right) & \geq v_{2}\left(F_{1}\right)+v_{2}\left(F_{1}\right)+\frac{1}{2} v_{2}\left(A_{1}\right) \\
& >\frac{1}{8} \boldsymbol{\mu}_{2}+\frac{1}{8} \boldsymbol{\mu}_{2}+\frac{1}{2}\left(3 \boldsymbol{\mu}_{2}-\frac{7}{8} \boldsymbol{\mu}_{2}-\frac{7}{8} \boldsymbol{\mu}_{2}\right)=\frac{7}{8} \boldsymbol{\mu}_{2} .
\end{aligned}
$$

Now, if $v_{2}\left(C_{1}\right) \geq \frac{7}{8} \boldsymbol{\mu}_{2}$ then (i) of Claim 5.3 implies that $\min \left\{v_{2}\left(C_{1}\right), v_{2}\left(A_{3} \cup C_{2}\right)\right\} \geq \frac{7}{8} \boldsymbol{\mu}_{2}$. Similarly, if $v_{2}\left(F_{3}\right)+v_{2}\left(C_{2}\right) \geq \frac{7}{8} \boldsymbol{\mu}_{2}$ then (ii) of Claim 5.3 implies that $\min \left\{v_{2}\left(F_{1} \cup F_{2} \cup\right.\right.$ $\left.\left.C_{1}\right), v_{2}\left(F_{3} \cup C_{2}\right)\right\} \geq \frac{7}{8} \boldsymbol{\mu}_{2}$. In both cases, we have $\boldsymbol{\mu}_{2}\left(2, A_{1} \cup A_{3}\right) \geq \frac{7}{8} \boldsymbol{\mu}_{2}$. So, it is left to examine the case where both $v_{2}\left(C_{1}\right)$ and $v_{2}\left(F_{3}\right)+v_{2}\left(C_{2}\right)$ are less than $\frac{7}{8} \boldsymbol{\mu}_{2}$. 
Claim 5.4. Let $C_{1}, C_{2}, A_{3}, F_{1}, F_{2}, F_{3}$ be as above and $\max \left\{v_{2}\left(C_{1}\right), v_{2}\left(F_{3} \cup C_{2}\right)\right\}<$ $\frac{7}{8} \boldsymbol{\mu}_{2}$. Then $\min \left\{v_{2}\left(F_{1} \cup C_{1}\right), v_{2}\left(F_{2} \cup F_{3} \cup C_{2}\right)\right\} \geq \frac{7}{8} \boldsymbol{\mu}_{2}$.

PROof. Recall that $v_{2}\left(A_{1}\right)+v_{2}\left(A_{3}\right)>\frac{17}{8} \boldsymbol{\mu}_{2}$. Suppose $v_{2}\left(F_{1} \cup C_{1}\right)<\frac{7}{8} \boldsymbol{\mu}_{2}$. Then $v_{2}\left(F_{2} \cup\right.$ $\left.F_{3} \cup C_{2}\right)>\frac{10}{8} \boldsymbol{\mu}_{2}$. Since $v_{2}\left(F_{3} \cup C_{2}\right)<\frac{7}{8} \boldsymbol{\mu}_{2}$ we have $v_{2}\left(F_{2}\right)>\frac{3}{8} \boldsymbol{\mu}_{2}$. But then we get the contradiction

$$
\frac{7}{8} \boldsymbol{\mu}_{2}>v_{2}\left(A_{3}\right)=v_{2}\left(F_{1}\right)+v_{2}\left(F_{2}\right)+v_{2}\left(F_{3}\right) \geq \frac{1}{8} \boldsymbol{\mu}_{2}+\frac{3}{8} \boldsymbol{\mu}_{2}+\frac{3}{8} \boldsymbol{\mu}_{2}=\frac{7}{8} \boldsymbol{\mu}_{2} .
$$

Hence, $v_{2}\left(F_{1} \cup C_{1}\right) \geq \frac{7}{8} \boldsymbol{\mu}_{2}$. Similarly, suppose $v_{2}\left(F_{2} \cup F_{3} \cup C_{2}\right)<\frac{7}{8} \boldsymbol{\mu}_{2}$. Then $v_{2}\left(F_{1} \cup C_{1}\right)>$ $\frac{10}{8} \boldsymbol{\mu}_{2}$. Since $v_{2}\left(C_{1}\right)<\frac{7}{8} \boldsymbol{\mu}_{2}$ we have $v_{2}\left(F_{1}\right)>\frac{3}{8} \boldsymbol{\mu}_{2}$. Then we get the contradiction

$$
\frac{7}{8} \boldsymbol{\mu}_{2}>v_{2}\left(A_{3}\right)=v_{2}\left(F_{1}\right)+v_{2}\left(F_{2}\right)+v_{2}\left(F_{3}\right) \geq \frac{3}{8} \boldsymbol{\mu}_{2}+\frac{3}{8} \boldsymbol{\mu}_{2}+\frac{3}{8} \boldsymbol{\mu}_{2}=\frac{9}{8} \boldsymbol{\mu}_{2} .
$$

Hence, $v_{2}\left(F_{2} \cup F_{3} \cup C_{2}\right) \geq \frac{7}{8} \boldsymbol{\mu}_{2}$.

Claim 5.4 implies $\boldsymbol{\mu}_{2}\left(2, A_{1} \cup A_{3}\right) \geq \frac{7}{8} \boldsymbol{\mu}_{2}$ and this concludes the proof.

5.2. Values in $\{0,1,2\}$

Bouveret and Lemaître [2016] consider a binary setting where all valuation functions take values in $\{0,1\}$, i.e., for each $i \in N$, and $j \in M, v_{i j} \in\{0,1\}$. This can correspond to expressing approval or disapproval for each item. It is then shown that it is always possible to find a maximin share allocation in polynomial time. In fact, they show that the Greedy Round-Robin algorithm, presented in Section 3, computes such an allocation in this case.

Here, we extend this result to the setting where each $v_{i j}$ is in $\{0,1,2\}$, allowing the agents to express two types of approval for the items. Enlarging the set of possible values from $\{0,1\}$ to $\{0,1,2\}$ by just one extra possible value makes the problem significantly more complex. Greedy Round-Robin does not work in this case, so a different algorithm is developed.

THEOREM 5.5. Let $N=[n]$ be a set of agents and $M=[m]$ be a set of items. If for any $i \in N$, agent $i$ has a valuation function $v_{i}$ such that $v_{i j} \in\{0,1,2\}$ for any $j \in M$, then we can find, in time $O(n m \log m)$, an allocation $\left(T_{1}, \ldots, T_{n}\right)$ of $M$ so that $v_{i}\left(T_{i}\right) \geq \boldsymbol{\mu}_{i}(n, M)$ for every $i \in[n]$.

To design our algorithm, we make use of an important observation by Bouveret and Lemaitre [2016] that allows us to reduce appropriately the space of valuation functions that we are interested in. We say that the agents have fully correlated valuation functions if they agree on a common ranking of the items in decreasing order of values. That is, $\forall i \in N$, if $M=\{1,2, \ldots, m\}$, we have $v_{i 1} \geq v_{i 2} \geq \ldots \geq v_{i m}$. In Bouveret and Lemaitre [2016], the authors show that to find a maximin share allocation for any set of valuation functions, it suffices to do so in an instance where the valuation functions are fully correlated. This family of instances seems to be the difficulty in computing such allocations. Actually, their result preserves approximation ratios as well (with the same proof); hence we state this stronger version. For a valuation function $v_{i}$ let $\sigma_{i}$ be a permutation on the items such that $v_{i}\left(\sigma_{i}(j)\right) \geq v_{i}\left(\sigma_{i}(j+1)\right)$ for $j \in\{1, \ldots, m-1\}$. We denote the function $v_{i}\left(\sigma_{i}(\cdot)\right)$ by $v_{i}^{\uparrow}$. Note that $v_{1}^{\uparrow}, v_{2}^{\uparrow}, \ldots, v_{n}^{\uparrow}$ are now fully correlated.

THEOREM 5.6 ([Bouveret and Lemaitre 2016]). Let $N=[n]$ be a set of agents with additive valuation functions, $M=[m]$ be a set of goods and $\rho \in(0,1]$. Given an allocation $\left(T_{1}, \ldots, T_{n}\right)$ of $M$ so that $v_{i}^{\uparrow}\left(T_{i}\right) \geq \rho \boldsymbol{\mu}_{i}(n, M)$ for every $i$, one can produce in linear time an allocation $\left(T_{1}^{\prime}, \ldots, T_{n}^{\prime}\right)$ of $M$ so that $v_{i}\left(T_{i}^{\prime}\right) \geq \rho \boldsymbol{\mu}_{i}(n, M)$ for every $i$. 
We are ready to state a high level description of our algorithm. The detailed description, however, is deferred to the end of this subsection. The reason for this is that the terminology needed is gradually introduced through a series of lemmas motivating the idea behind the algorithm and proving its correctness. In fact, the remainder of the subsection is the proof of Theorem 5.5. Algorithm 6 in the end summarizes all the steps.

Algorithm Outline: We first construct $v_{1}^{\uparrow}, v_{2}^{\uparrow}, \ldots, v_{n}^{\uparrow}$ and work with them instead. The Greedy Round-Robin algorithm may not directly work, but we partition the items in a similar fashion, although without giving them to the agents. Then, we show that it is possible to choose some subsets of items and redistribute them in a way that guarantees that everyone can get a bundle of items with enough value. At a higher level, we could say that the algorithm simulates a variant of the Greedy Round-Robin, where for an appropriately selected set of rounds the agents choose in the reverse order. Finally, a maximin share allocation can be obtained for the original $v_{i} \mathrm{~s}$, as described in Bouveret and Lemaître [2016].

Proof of TheOREM 5.5. According to Theorem 5.6 it suffices to focus on instances where the valuation functions take values in $\{0,1,2\}$ and are fully correlated. Given such an instance we distribute the $m$ objects into $n$ buckets in decreasing order, i.e., bucket $i$ will get items $i, n+i, 2 n+i, \ldots$. Notice that this is compatible with how the Greedy Round-Robin algorithm could distribute the items; however, we do not assign any buckets to any agents yet. We may assume that $m=k n$ for some $k \in \mathbb{N}$; if not, we just add a few extra items with 0 value to everyone. It is convenient to picture the collection of buckets as the matrix

$$
B=\left(\begin{array}{cccc}
(k-1) n+1 & (k-1) n+2 & \cdots & k n \\
\vdots & \vdots & \ddots & \vdots \\
n+1 & n+2 & \cdots & 2 n \\
1 & 2 & \cdots & n
\end{array}\right)
$$

since our algorithm will systematically redistribute groups of items corresponding to rows of $B$.

Before we state the algorithm, we establish some properties regarding these buckets and the way each agent views the values of these bundles. First, we introduce some terminology.

\section{Definition 5.7. We say that agent $i$ is}

- satisfied with respect to the current buckets, if all the buckets have value at least $\boldsymbol{\mu}_{i}(n, M)$ according to $v_{i}$.

- left-satisfied with respect to the current buckets, if she is not satisfied, but at least the $n / 2$ leftmost buckets have value at least $\boldsymbol{\mu}_{i}(n, M)$ according to $v_{i}$.

- right-satisfied if the same as above hold, but for the rightmost $n / 2$ buckets.

Now suppose that we see agent $i$ 's view of the values in the buckets. A typical view would have the following form (recall the goods are ranked from highest to lowest 
value):

$$
\left(\begin{array}{cccccccccc}
0 & 0 & 0 & 0 & 0 & 0 & \cdots & 0 & 0 & 0 \\
\cdot & \cdot & \cdot & \cdot & \cdot & \cdot & \cdots & \cdot & \cdot & \cdot \\
1 & 1 & 1 & 1 & 1 & 0 & \cdots & 0 & 0 & 0 \\
\cdot & \cdot & \cdot & \cdot & \cdot & \cdot & \cdots & \cdot & \cdot & \cdot \\
1 & 1 & 1 & 1 & 1 & 1 & \cdots & 1 & 1 & 1 \\
2 & 2 & 2 & 1 & 1 & 1 & \cdots & 1 & 1 & 1 \\
\cdot & \cdot & \cdot & \cdot & \cdot & \cdot & \cdots & \cdot & \cdot & \cdot \\
2 & 2 & 2 & 2 & 2 & 2 & \cdots & 2 & 2 & 2
\end{array}\right)
$$

A row that has only $2 \mathrm{~s}$ for $i$ will be called a 2-row for $i$. A row that has both $2 \mathrm{~s}$ and $1 \mathrm{~s}$ will be called a 2/1-row for $i$, and so forth. An agent can also have a 2/1/0-row. It is not necessary, of course, that an agent will have all possible types of rows in her view. Note, however, that there can be at most one 1/0-row and at most one 2/1-row in her view. We first prove the following lemma for agents that are not initially satisfied.

LEMMA 5.8. Any agent not satisfied with respect to the initial buckets must have both a 1/0-row and a 2/1-row in her view of B. Moreover, initially all agents are either satisfied or left-satisfied.

Proof. Let us focus on the multiset of values of an agent that is not satisfied, say $i$. It is straightforward to see that if $i$ has no $1 \mathrm{~s}$, or the number of $2 \mathrm{~s}$ is a multiple of $n$ (including 0 ), then agent $i$ gets value $\boldsymbol{\mu}_{i}(n, M)$ from any bucket. So, $i$ must have a row with both $2 \mathrm{~s}$ and $1 \mathrm{~s}$. If this is a $2 / 1 / 0$-row, then again it is easy to see that the initial allocation is a maximin share allocation for $i$. So, $i$ has a 2/1-row. The only case where she does not have a 1/0-row is if the total number of $1 \mathrm{~s}$ and $2 \mathrm{~s}$ is a multiple of $n$.But then the maximum and the minimum value of the initial buckets differ by 1 , hence we have a maximin share allocation and $i$ is satisfied.

Next we show that an agent $i$ who is not initially satisfied is left-satisfied. In what follows we only refer to $i$ 's view. Buckets $B_{1}$ and $B_{n}$, indexed by the corresponding columns of $B$, have maximum and minimum total value respectively. Since $i$ is not satisfied, we have $v_{i}\left(B_{1}\right) \geq v_{i}\left(B_{n}\right)+2$, but the way we distributed the items guarantees that the difference between any two buckets is at most the largest value of an item; so $v_{i}\left(B_{1}\right)=v_{i}\left(B_{n}\right)+2$. Moreover, since $v_{i}(M) \geq n \boldsymbol{\mu}_{i}(n, M)$ and $v_{i}\left(B_{n}\right)<\boldsymbol{\mu}_{i}(n, M)$, we must have $v_{i}\left(B_{1}\right)>\boldsymbol{\mu}_{i}(n, M)$. This implies that $v_{i}\left(B_{1}\right)=\boldsymbol{\mu}_{i}(n, M)+1$ and $v_{i}\left(B_{n}\right)=$ $\boldsymbol{\mu}_{i}(n, M)-1$.

More generally, we have buckets of value $\boldsymbol{\mu}_{i}(n, M)+1$ (leftmost columns), we have buckets of value $\boldsymbol{\mu}_{i}(n, M)-1$ (rightmost columns), and maybe some other buckets of value $\boldsymbol{\mu}_{i}(n, M)$ (columns in the middle). We know that the total value of all the items is at least $n \boldsymbol{\mu}_{i}(n, M)$, so, by summing up the values of the buckets, we conclude that there must be at most $n / 2$ buckets of value $\boldsymbol{\mu}_{i}(n, M)-1$. Therefore $i$ is left-satisfied.

So far we may have some agents that could take any bucket and some agents that would take any of the $n / 2$ (at least) first buckets. Clearly, if the left-satisfied agents are at most $n / 2$ then we can easily find a maximin share allocation. However, there is no guarantee that there are not too many left-satisfied agents initially, so we try to fix this by reversing some of the rows of $B$. To make this precise, we say that we reverse the ith row of $B$ when we take items $(i-1) n+1,(i-1) n+2, \ldots$, in and we put item $i n$ in bucket 1 , item in -1 in bucket 2 , etc.

The algorithm then proceeds by picking a subset of rows of $B$ and reversing them. The rows are chosen appropriately so that the resulting buckets (i.e., the columns of $B$ ) can be easily paired with the agents to get a maximin share allocation. First, it is crucial to understand the effect that the reversal of a set of rows has to an agent. 
LEMMA 5.9. Any agent satisfied with respect to the initial buckets remains satisfied independently of the rows of $B$ that we may reverse. On the other hand, any agent not satisfied with respect to the initial buckets, say agent $i$, is affected if we reverse her $1 / 0$ row or her 2/1-row. If we reverse only one of those, then $i$ becomes satisfied with respect to the new buckets; if we reverse both, then i becomes right-satisfied. The reversal of any other rows is irrelevant to agent $i$.

Proof. Fix an agent $i$. First notice that, due to symmetry, reversing any row that for $i$ is a 2-row, a 1-row, or a 0 -row does not improve or worsen the initial allocation from $i$ 's point of view. Also, clearly, reversing both the 1/0-row and the 2/1-row of a leftsatisfied agent makes her right-satisfied. Similarly, if $i$ is satisfied and has a 2/1/0-row, or has a 2/1-row but no 1/0-row, or has a 1/0-row but no 2/1-row, then reversing those keeps $i$ satisfied.

The interesting case is when $i$ has both a 1/0-row and a 2/1-row. If $i$ is satisfied, then even removing her 1/0-row leaves all the buckets with at least as much value as the last bucket; so reversing it keeps $i$ satisfied. A similar argument holds for $i$ 's 2/1-row as well. If $i$ is not satisfied, then the difference of the values of the first and the last bucket will be 2 . Like in the proof of Lemma 5.8, the number of columns that have 1 in $i$ 's $1 / 0$-row and 2 in $i$ 's $2 / 1$-row (i.e., total value $\boldsymbol{\mu}_{i}(n, M)+1$ ) are at least as many as the columns that have 0 in $i$ 's $1 / 0$-row and 1 in $i$ 's $2 / 1$-row (i.e., total value $\boldsymbol{\mu}_{i}(n, M)-1$ ). So, by reversing her 1/0-row, the values of all the "worst" (rightmost) buckets increase by 1 , the values of some of the "best" (leftmost) buckets decrease by 1 , and the values of the buckets in the middle either remain the same or increase by 1 . The difference between the best and the worst buckets now is 1 (at most), so this is a maximin share allocation for $i$ and she becomes satisfied. Due to symmetry, the same holds for reversing $i$ 's 2/1row only.

Now, what Lemma 5.9 guarantees is that when we reverse some of the rows of the initial $B$, we are left with agents that are either satisfied, left-satisfied, or rightsatisfied. If the rows are chosen so that there are at most $n / 2$ left-satisfied and at most $n / 2$ right-satisfied agents, then there is an obvious maximin share allocation: to any left-satisfied agent we arbitrarily give one of the first $n / 2$ buckets, to any rightsatisfied agent we arbitrarily give one of the last $n / 2$ buckets, and to each of the remaining agents we arbitrarily give one of the remaining buckets. In Lemma 5.10 below, we prove that it is easy to find which rows to reverse to achieve that.

We use a graph theoretic formulation of the problem for clarity. With respect to the initial buckets, we define a graph $G=(V, E)$ with $V=[k]$, i.e., $G$ has a vertex for each row of $B$. Also, for each left-satisfied agent $i, G$ has an edge connecting $i$ 's 1/0-row and 2/1-row. We delete, if necessary, any multiple edges to get a simple graph with $n$ edges at most. We want to color the vertices of $G$ with two colors, "red" (for reversed rows) and "blue" (for non reversed), so that the number of edges having both endpoints red is at most $n / 2$ and at the same time the number of edges having both endpoints blue is at most $n / 2$. Note that if we reverse the rows that correspond to red vertices, then the agents with red endpoints become right-satisfied, the agents with blue endpoints remain left-satisfied and the agents with both colors become satisfied. Moreover, the initially satisfied agents are not affected, and we can find a maximin share allocation as previously discussed. This is illustrated in Figure 3 below.

LEMMA 5.10. Given graph $G$ defined above, in time $O(k+n)$ we can color the vertices with two colors, red and blue, so that the number of edges with two red endpoints is less than $n / 2$ and the number of edges with two blue endpoints is at most $n / 2$.

PROOF. We start with all the vertices colored blue, and we arbitrarily recolor vertices red, one at a time, until the number of edges with two blue endpoints becomes 


$\begin{array}{ccc}0 & 0 & 0 \\ 0 & 0 & 0 \\ 1 & 1 & 0 \\ 2 & 1 & 1\end{array}$

left-satisfied

$\begin{array}{llll}0 & 0 & 0 \\ 1 & 0 & 0 & 1 \\ 1 & 1 & 1 \\ 2 & 2 & 2\end{array}$

satisfied

\begin{tabular}{ccc}
1 & 1 & 0 \\
1 & 1 & 1 \\
2 & 2 & 1 \\
2 & 2 & 2 \\
\hdashline & left-satisfied
\end{tabular}

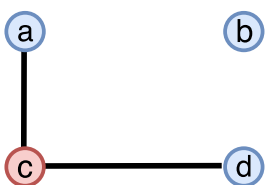

(b)

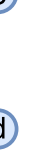

$\begin{array}{lll}0 & 0 & 0 \\ 0 & 0 & 0 \\ 0 & 1 & 1\end{array}$

satisfied (b)

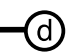

row a

row b

row C

row d

\begin{tabular}{ccccl}
1 & 1 & 0 & row a \\
1 & 1 & 1 & row b \\
1 & 2 & 2 & row c reversed \\
2 & 2 & 2 & row d \\
\hdashline & 5 &
\end{tabular}

Fig. 3. Assuming an instance with 3 agents and 11 items, the tables on top are the three different views on the initial buckets. This results in the graph shown in the middle-before and after the coloring. By reversing row $c$ that corresponds to a red vertex, every agent becomes satisfied and thus any matching of the columns to the agents defines an MMS allocation.

at most $|E| / 2$ for the first time. Assume this happens after recoloring vertex $u$. Before turning $u$ from blue to red, the number of edges with at most one blue endpoint was strictly less than $|E| / 2$. Also, the recoloring of $u$ did not force any of the edges with two blue endpoints to become edges with two red endpoints. So, the number of edges with two red endpoints after the recoloring of $u$ is at most equal to the number of edges with at most one blue endpoint before the recoloring of $u$, i.e., less than $|E| / 2$. To complete the proof, notice that $|E| \leq n$. For the running time, notice that each vertex changes color at most once and when this happens we only need to examine the adjacent vertices in order to update the counters on each type of edges (only red, only blue, or both).

Lemma 5.10 completes the proof of correctness for Algorithm 6 that is summarized below. For the running time notice that $v_{1}^{\uparrow}, \ldots, v_{n}^{\uparrow}$ can be computed in $O(n m \log m)$, since we get $v_{i}^{\uparrow}$ by sorting $v_{i 1}, \ldots, v_{i m}$. Also step 5 can be computed in $O(n m)$; for each agent $i$ we scan the first column of $B$ to find her (possible) 1/0-row and 2/1-row, and then in $O(n)$ we check whether she is left-satisfied by checking that the positions that have 1 in $i$ 's $1 / 0$-row and 2 in $i$ 's 2/1-row are at least as many as the positions that have 0 in $i$ 's $1 / 0$-row and 1 in $i$ 's $2 / 1$-row. 


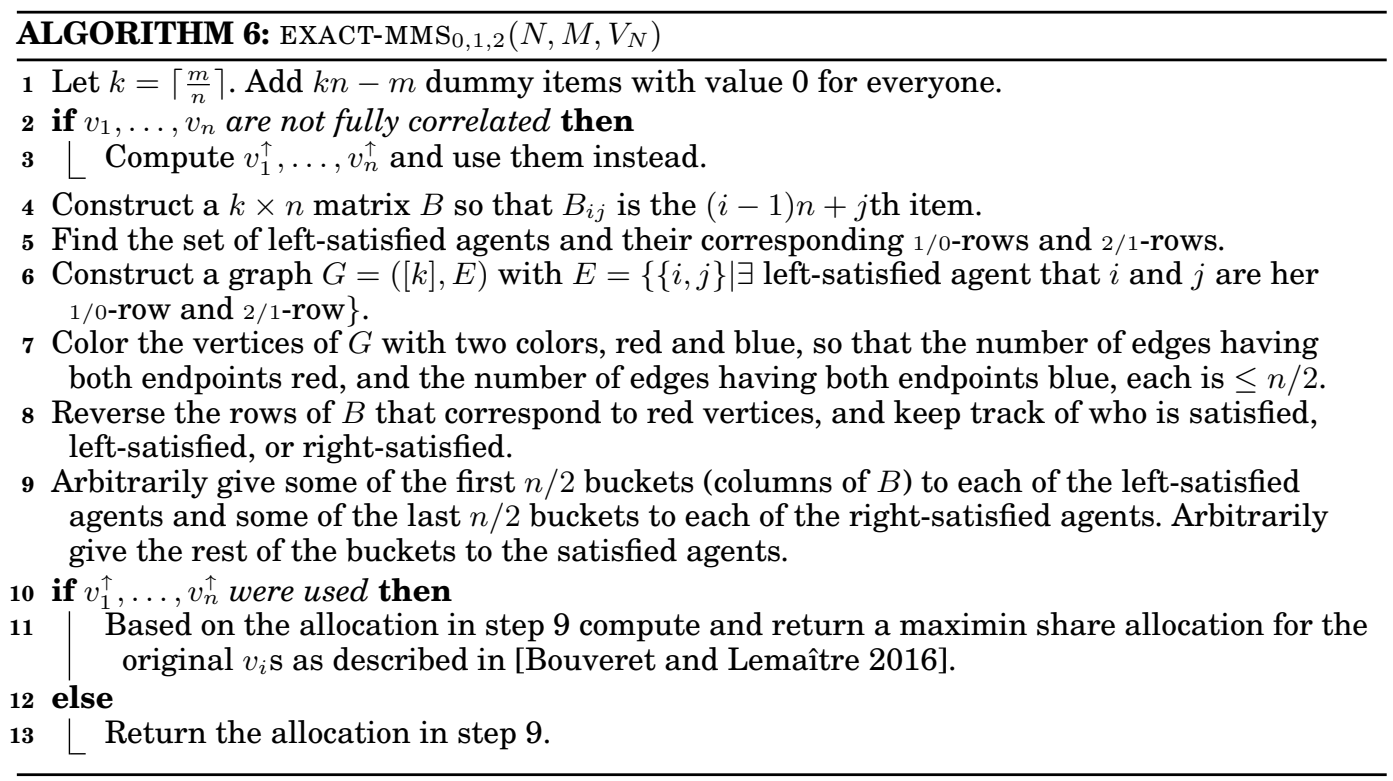

\section{A PROBABILISTIC ANALYSIS}

As argued in the previous works [Bouveret and Lemaitre 2016; Procaccia and Wang 2014], it has been quite challenging to prove impossibility results. Setting efficient computation aside, what is the best $\rho$ for which a $\rho$-approximate allocation does exist? All we know so far is that $\rho \neq 1$ by the elaborate constructions by Kurokawa et al. [2016], and Procaccia and Wang [2014]. However, extensive experimentation by Bouveret and Lemaître [2016] (and also by Procaccia and Wang [2014]), showed that in all generated instances, there always existed a maximin share allocation. Motivated by these experimental observations and by the lack of impossibility results, we present a probabilistic analysis, showing that indeed we expect that in most cases there exist allocations where every agent receives her maximin share. In particular, we analyze the Greedy Round-Robin algorithm from Section 3 when each $v_{i j}$ is drawn from the uniform distribution over $[0,1]$.

Recently, Kurokawa et al. [2016] show similar results for a large set of distributions over $[0,1]$, including $U[0,1]$. Although, asymptotically, their results yield a theorem that is more general than ours, we consider our analysis to be of independent interest, since we have much better bounds on the probabilities for the special case of $U[0,1]$, even for relatively small values of $n$.

For completeness, before stating and proving our results, we include the version of Hoeffding's inequality we are going to use.

THEOREM 6.1 ([Hoeffding 1963]). Let $X_{1}, X_{2}, \ldots, X_{n}$ be independent random variables with $X_{i} \in[0,1]$ for $i \in[n]$. Then for the empirical mean $\bar{X}=\frac{1}{n}\left(X_{1}+\ldots+X_{n}\right)$ we have $\mathrm{P}(\bar{X}-\mathrm{E}[\bar{X}] \geq t) \leq \exp \left(-2 n t^{2}\right)$.

We start with Theorem 6.2. Its proof is based on tools like Hoeffding's and Chebyshev's inequalities, and on a careful estimation of the probabilities when $m<3 n$. Note that for $m \geq 2 n$, the theorem provides an even stronger guarantee than the maximin share (by Claim 3.1). 
THEOREM 6.2. Let $N=[n]$ be a set of agents and $M=[m]$ be a set of goods, and assume that the $v_{i j}$ s are i.i.d. random variables that follow $U[0,1]$. Then, for $m \geq 2 n$ and large enough $n$, the Greedy Round-Robin algorithm allocates to each agent $i$ a set of goods of total value at least $\frac{1}{n} \sum_{j=1}^{m} v_{i j}$ with probability $1-o(1)$. The o(1) term is $O(1 / n)$ when $m>2 n$ and $O(\log n / n)$ when $m=2 n$.

PROOF. In what follows we assume that agent 1 chooses first, agent 2 chooses second, and so forth. We consider several cases for the different ranges of $m$. We first assume that $2 n \leq m<3 n$.

It is illustrative to consider the case of $m=2 n$ and examine the $n$th agent that chooses last. Like all the agents in this case, she receives exactly two items; let $Y_{n}$ be the total value of those items. From her perspective, she sees $n+1$ values chosen uniformly from $[0,1]$, picks the maximum of those, then u.a.r. $n-1$ of the rest are removed, and she takes the last one as well. If we isolate this random experiment, it is as if we take $Y_{n}=\max \left\{X_{1}, \ldots, X_{n+1}\right\}+X_{Y}$, where $Y \sim U(\{1,2, \ldots, n+1\} \backslash\{\mu\})$, $\mu \in \arg \max \left\{X_{1}, \ldots, X_{n+1}\right\}, X_{i} \sim U[0,1] \forall i \in[n+1]$, and all the $X_{i}$ s are independent. We estimate now the probability $\mathrm{P}\left(Y_{n} \leq a\right)$ for $1<a<2$. We will set $a$ to a particular value in this interval later on. In fact, we bound this probability using the corresponding probability for $Z_{n}=\max \left\{X_{1}, \ldots, X_{n+1}\right\}+X_{Y^{\prime}}$, where $Y^{\prime} \sim U\{1,2, \ldots, n+1\}$. For $Z_{n}$ we have

$$
\begin{aligned}
\mathrm{P}\left(Z_{n} \leq a\right)= & \sum_{i=1}^{n+1} \int_{0}^{a} \mathrm{P}\left(\max _{1 \leq j \leq n+1} X_{j} \leq t \wedge Y=i \wedge X_{i} \leq a-t\right) d t \\
= & (n+1) \int_{0}^{a} \mathrm{P}\left(\max _{1 \leq j \leq n+1} X_{j} \leq t \wedge Y=1 \wedge X_{1} \leq a-t\right) d t \\
= & \int_{0}^{a} \mathrm{P}\left(\max _{1 \leq j \leq n+1} X_{j} \leq t \wedge X_{1} \leq a-t\right) d t \\
= & \int_{0}^{a} \mathrm{P}\left(X_{1} \leq t \wedge X_{1} \leq a-t \wedge X_{2} \leq t \wedge \ldots \wedge X_{n+1} \leq t\right) d t \\
= & \int_{0}^{a / 2} \mathrm{P}\left(X_{1} \leq t \wedge X_{2} \leq t \wedge \ldots \wedge X_{n+1} \leq t\right) d t+ \\
& +\int_{a / 2}^{1} \mathrm{P}\left(X_{1} \leq a-t \wedge X_{2} \leq t \wedge \ldots \wedge X_{n+1} \leq t\right) d t+ \\
& +\int_{1}^{a} \mathrm{P}\left(X_{1} \leq a-t \wedge X_{2} \leq t \wedge \ldots \wedge X_{n+1} \leq t\right) d t \\
= & \int_{0}^{a / 2} t^{n+1} d t+\int_{a / 2}^{1}(a-t) t^{n} d t+\int_{1}^{a}(a-t) d t .
\end{aligned}
$$

Also, by the definition of $Y^{\prime}$ we have $\mathrm{P}\left(Y^{\prime} \notin \arg \max \left\{X_{1}, \ldots, X_{n+1}\right\}\right)=n /(n+1)$. Therefore, for $Y_{n}$ we get

$$
\begin{aligned}
\mathrm{P}\left(Y_{n} \leq a\right) & =\mathrm{P}\left(Z_{n} \leq a \mid Y^{\prime} \notin \arg \max \left\{X_{1}, \ldots, X_{n+1}\right\}\right) \\
& =\frac{\mathrm{P}\left(Z_{n} \leq a \wedge Y^{\prime} \notin \arg \max \left\{X_{1}, \ldots, X_{n+1}\right\}\right)}{\mathrm{P}\left(Y^{\prime} \notin \arg \max \left\{X_{1}, \ldots, X_{n+1}\right\}\right)} \\
& \leq \frac{\mathrm{P}\left(Z_{n} \leq a\right)}{\mathrm{P}\left(Y^{\prime} \notin \arg \max \left\{X_{1}, \ldots, X_{n+1}\right\}\right)}=\frac{n+1}{n} \mathrm{P}\left(Z_{n} \leq a\right) \\
& =\frac{n+1}{n}\left(\int_{0}^{a / 2} t^{n+1} d t+\int_{a / 2}^{1}(a-t) t^{n} d t+\int_{1}^{a}(a-t) d t\right),
\end{aligned}
$$


where for the inequality we used the fact that $\mathrm{P}(A \cap B) \leq \mathrm{P}(A)$ for any events $A, B$.

A similar analysis for the $j$ th agent yields

$$
\mathrm{P}\left(Y_{j} \leq a\right) \leq \frac{2 n-j+1}{n}\left(\int_{0}^{a / 2} t^{2 n-j+1} d t+\int_{a / 2}^{1}(a-t)^{n-j+1} t^{n} d t+\int_{1}^{a}(a-t)^{n-j+1} d t\right) .
$$

In the more general case where $m=2 n+\kappa(n), 0 \leq \kappa(n)<n$, we have a similar calculation for the agents that receive only two items in the Greedy Round-Robin algorithm, as well as for the first two items of the first $\kappa(n)$ agents (who receive three items each). Let $Y_{i}$ be the total value agent $i$ receives, and $W_{i}$ be the value of her first two items. Of course, for the last $2 n$ players, $Y_{i}=W_{i}$. Also, recall that $\sum_{j=1}^{m} v_{i j}=v_{i}(M)$. We now relate the probability that we are interested in estimating, with the probabilities $\mathrm{P}\left(Y_{i} \leq a\right)$ that we have already bounded. We will then proceed by setting $\alpha$ appropriately. We have

$$
\begin{aligned}
\mathrm{P}\left(\exists i \text { such that } Y_{i}\right. & \left.<\frac{1}{n} \sum_{j=1}^{m} v_{i j}\right) \leq \sum_{i=1}^{n} \mathrm{P}\left(Y_{i}<\frac{v_{i}(M)}{n}\right) \\
& =\sum_{i=1}^{n} \mathrm{P}\left(Y_{i}<\min \left\{\frac{v_{i}(M)}{n}, a\right\} \vee \frac{v_{i}(M)}{n}>\max \left\{Y_{i}, a\right\}\right) \\
& \leq \sum_{i=1}^{n} \mathrm{P}\left(Y_{i}<\min \left\{\frac{v_{i}(M)}{n}, a\right\}\right)+\sum_{i=1}^{n} \mathrm{P}\left(\frac{v_{i}(M)}{n}>\max \left\{Y_{i}, a\right\}\right) \\
& \leq \sum_{i=1}^{n} \mathrm{P}\left(Y_{i}<a\right)+\sum_{i=1}^{n} \mathrm{P}\left(\frac{v_{i}(M)}{n}>a\right) .
\end{aligned}
$$

To upper bound the first sum we use the $W_{i}$ s, i.e., we do not take into account the third item that the first $\kappa(n)$ agents receive. By the definition of $Y_{i}, W_{i}$, for these first $\kappa(n)$ agents we have $\mathrm{P}\left(Y_{i}<a\right) \leq \mathrm{P}\left(W_{i}<a\right)$, while for the remaining agents we have $\mathrm{P}\left(Y_{i}<a\right)=\mathrm{P}\left(W_{i}<a\right)$. Note that the bounds for $\mathrm{P}\left(Y_{i} \leq a\right)$ calculated above, here hold for $\kappa(n)+1 \leq i \leq n$. For $1 \leq i \leq \kappa(n)$ the same bounds hold for $\mathrm{P}\left(W_{i} \leq a\right)$.

$$
\begin{aligned}
& \sum_{i=1}^{n} \mathrm{P}\left(Y_{i}<a\right) \leq \sum_{i=1}^{\kappa(n)} \mathrm{P}\left(W_{i}<a\right)+\sum_{i=\kappa(n)+1}^{n} \mathrm{P}\left(Y_{i}<a\right) \\
& \quad \leq \sum_{i=1}^{n} \frac{m-i+1}{n}\left(\int_{0}^{a / 2} t^{m-i+1} d t+\int_{a / 2}^{1}(a-t)^{n+\kappa(n)-i+1} t^{n} d t+\int_{1}^{a}(a-t)^{n+\kappa(n)-i+1} d t\right) \\
& \quad \leq 3 \sum_{j=1}^{n}\left(\int_{0}^{a / 2} t^{n+\kappa(n)+j} d t+\int_{a / 2}^{1}(a-t)^{\kappa(n)+j} t^{n} d t+\int_{1}^{a}(a-t)^{\kappa(n)+j} d t\right) \\
& \quad=3\left(\sum_{j=1}^{n} \frac{(a / 2)^{n+\kappa(n)+j+1}}{n+\kappa(n)+j+1}+\sum_{j=1}^{n} \int_{a / 2}^{1}(a-t)^{\kappa(n)+j} t^{n} d t+\sum_{j=1}^{n} \int_{0}^{a-1} u^{\kappa(n)+j} d u\right) .
\end{aligned}
$$

We are going to bound each sum separately. We set $a=1+\frac{\kappa(n)}{2 n}+\sqrt{\frac{3 \ln n}{n}}=\frac{m}{2 n}+\sqrt{\frac{3 \ln n}{n}}$. Note that for $n \geq 46$ we have $a \in(1,2)$. Consider the first sum:

$$
\sum_{j=1}^{n} \frac{(a / 2)^{n+\kappa(n)+j+1}}{n+\kappa(n)+j+1} \leq \frac{(a / 2)^{n+\kappa(n)+2}}{n+\kappa(n)+2} \sum_{i=0}^{n-1}(a / 2)^{i}
$$




$$
<\frac{1}{n+\kappa(n)+2} \cdot \frac{(a / 2)^{n+\kappa(n)+2}}{1-a / 2}=O(1 / n)
$$

where we got $O(1 / n)$ because the bound is at most $\frac{3}{n}$ for $n \geq 57$ and for any value of $\kappa(n)$.

Next, we deal with the second sum:

$$
\begin{aligned}
\sum_{j=1}^{n} \int_{a / 2}^{1}(a-t)^{\kappa(n)+j} t^{n} d t & <\int_{a / 2}^{1} t^{n}\left(\sum_{j=0}^{\infty}(a-t)^{\kappa(n)+j}\right) d t \leq \int_{a / 2}^{1} t^{n}(a / 2)^{\kappa(n)} \frac{1}{1-a+t} d t \\
& \leq \frac{(a / 2)^{\kappa(n)}}{1-a / 2} \int_{a / 2}^{1} t^{n} d t=\frac{(a / 2)^{\kappa(n)}}{1-a / 2}\left(\frac{1-(a / 2)^{n+1}}{n+1}\right)=O(1 / n)
\end{aligned}
$$

Here, for $n \geq 58$ the bound is at most $\frac{10}{n}$ for any $\kappa(n)$.

Finally, for the third sum, we rewrite it as

$$
\sum_{j=1}^{n} \int_{0}^{a-1} u^{\kappa(n)+j} d u=\sum_{j=1}^{n} \frac{(a-1)^{\kappa(n)+j+1}}{\kappa(n)+j+1}=\sum_{i=\kappa(n)+2}^{n+\kappa(n)+1} \frac{(a-1)^{i}}{i}
$$

We are going to bound each term separately. Consider the case where $\kappa(n) \geq 5 \sqrt{n}$. For $n \geq 64$, it can be shown that $\frac{1}{5}\left(\frac{\kappa(n)}{2 n}+\sqrt{\frac{3 \ln n}{n}}\right)^{5 \sqrt{n}}<\frac{10}{n^{3 / 2}}$. So,

$$
\sum_{i=\kappa(n)+2}^{n+\kappa(n)+1} \frac{(a-1)^{i}}{i} \leq \sum_{i=1}^{n} \frac{(a-1)^{\kappa(n)}}{\kappa(n)} \leq n \cdot \frac{\left(\frac{\kappa(n)}{2 n}+\sqrt{\frac{3 \ln n}{n}}\right)^{5 \sqrt{n}}}{5 \sqrt{n}} \leq n \cdot \frac{10}{n^{2}}=\frac{10}{n}
$$

On the other hand, when $\kappa(n)<5 \sqrt{n}$, we have $a-1<\frac{2.5+\sqrt{3 \ln n}}{\sqrt{n}}$. For $n \geq 59$ and $j \geq 10$ it can be shown that $\frac{1}{j}\left(\frac{2.5+\sqrt{3 \ln n}}{\sqrt{n}}\right)^{j}<\frac{30}{n^{2}}$. Of course, for $3 \leq j \leq 9$ it is true that $\frac{1}{j}\left(\frac{2.5+\sqrt{3 \ln n}}{\sqrt{n}}\right)^{j}=o(1 / n)$, and particularly for $n \geq 59$ the sum $\sum_{i=3}^{9} \frac{1}{j}\left(\frac{2.5+\sqrt{3 \ln n}}{\sqrt{n}}\right)^{j}$ is bounded by $\frac{25}{n}$. In general, it is to be expected to have relatively large hidden constants when $m$ is very close to $2 n$. This changes quickly though; when $\kappa(n)>21$ the whole sum is less than $1 / n$. In any case, if $\kappa(n)>0$

$$
\begin{aligned}
\sum_{i=\kappa(n)+2}^{n+\kappa(n)+1} \frac{(a-1)^{i}}{i} & \leq \sum_{i=3}^{n+2} \frac{(a-1)^{i}}{i} \leq \sum_{i=3}^{9} \frac{1}{i}\left(\frac{2.5+\sqrt{3 \ln n}}{\sqrt{n}}\right)^{i}+\sum_{i=10}^{n+2} \frac{1}{i}\left(\frac{2.5+\sqrt{3 \ln n}}{\sqrt{n}}\right)^{i} \leq \\
& \leq O(1 / n)+(n-7) \frac{30}{n^{2}}=O(1 / n) .
\end{aligned}
$$

However, if $\kappa(n)=0$, we have

$$
\sum_{i=2}^{n+1} \frac{(a-1)^{i}}{i}=\left(\frac{2.5+\sqrt{3 \ln n}}{\sqrt{n}}\right)^{2}+\sum_{i=3}^{n+1} \frac{(a-1)^{i}}{i}=O\left(\frac{\log n}{n}\right) .
$$

So far, we have $\sum_{i=1}^{n} \mathrm{P}\left(Y_{i}<a\right)=O(1 / n)$ (or $O(\log n / n)$ when $\left.m=2 n\right)$. In order to complete the proof for this case we use Hoeffding's inequality to bound the probability 
that the average of the values for any agent is too large.

$$
\begin{aligned}
\sum_{i=1}^{n} \mathrm{P}\left(\frac{v_{i}(M)}{n}>a\right) & \leq n \cdot \mathrm{P}\left(\frac{v_{1}(M)}{n}>a\right)=n \cdot \mathrm{P}\left(\frac{v_{1}(M)}{m}>\frac{n}{m}\left(\frac{m}{2 n}+\sqrt{\frac{3 \ln n}{n}}\right)\right) \\
& =n \cdot \mathrm{P}\left(\frac{v_{1}(M)}{m}-\frac{1}{2}>\frac{n}{m} \sqrt{\frac{3 \ln n}{n}}\right) \leq n \cdot e^{-2 m\left(\frac{n}{m} \sqrt{\frac{3 \ln n}{n}}\right)^{2}} \\
& =n \cdot e^{-\frac{n}{m} \cdot 3 \ln n} \leq n \cdot e^{-2 \ln n}=\frac{1}{n} .
\end{aligned}
$$

Hence,

$$
\mathrm{P}\left(\exists i \text { such that } Y_{i}<\frac{v_{i}(M)}{n}\right)=\left\{\begin{array}{ll}
O\left(\frac{\log n}{n}\right) & \text { if } m=2 n \\
O\left(\frac{1}{n}\right) & \text { if } 2 n<m<3 n
\end{array} .\right.
$$

The remaining cases are for $m \geq 3 n$. We give the proof for $m \geq 4 n$. The cases for $3 n \leq m<3.5 n$ and $3.5 n \leq m<4 n$ differ in small details but they essentially follow the same analysis. We briefly discuss these cases at the end of the proof.

Assume that $k n \leq m<(k+1) n, k \geq 4$. We focus on the agent that choses last, i.e., agent $n$, who has the smallest expected value. She gets exactly $k$ items, and like before let $Y_{n}$ be the total value she receives. In order to bound $\mathrm{P}\left(Y_{n}<\beta\right)$ we introduce the random variables $Z_{n}$ and $W_{n}$. Consider the following random experiment involving the independent random variables $X_{1}, \ldots, X_{m-n+1}, X_{i} \sim U[0,1] \forall i \in[m-n+1]$. Given a realization of the $X_{i}$ s, i.e., some values $x_{1}, \ldots, x_{m-n+1}$ in $[0,1], Z_{n}$ is defined similarly to $Y_{n}$ :

- Initially, $Z_{n}=0$.

- While there are still $x_{i}$ s left, take the maximum of the remaining $x_{i} \mathrm{~s}$, add it to $Z_{n}$, remove it from the available numbers, and then remove the $x_{i}$ s with the $n-1$ highest indices.

- Return $Z_{n}$.

On the other hand, $W_{n}=\sum_{i=1}^{k-1} X_{(m+1-i n, m-i(n-1))}$, where $X_{(j, t)}$ is the $j$ th order statistic of $X_{1}, \ldots, X_{t}$. That is, $W_{n}$ is defined as the sum of the largest of all $x_{i}$ s, the second largest of the first $m-n+1 x_{i} \mathrm{~s}$, the third largest of the first $m-2 n+2 x_{i} \mathrm{~s}$, and so on.

It is not hard to see that always $W_{n} \leq Z_{n}$ (in fact, each term of $W_{n}$ is less than or equal to the corresponding term of $Z_{n}$ ) and that $Z_{n}$ follows the same distribution as $Y_{n}$. So, $\mathrm{P}\left(Y_{n}<\beta\right)=\mathrm{P}\left(Z_{n}<\beta\right) \leq \mathrm{P}\left(W_{n}<\beta\right)$. Using the fact that the $i$ th order statistic in a sample of size $\ell$ drawn independently from $U[0,1]$ has expected value $\frac{i}{\ell+1}$ and variance $\frac{i(\ell-i+1)}{(\ell+1)^{2}(\ell+2)}$ [Gentle 2009], we get

$$
\begin{aligned}
\mathrm{E}\left[W_{n}\right] & =\frac{m-n+1}{m-n+2}+\frac{m-2 n+1}{m-2 n+3}+\ldots+\frac{m-(k-1) n+1}{m-(k-1) n+k} \\
& \geq \frac{(k-1) n+1}{(k-1) n+2}+\frac{(k-2) n+1}{(k-2) n+3}+\ldots+\frac{n+1}{n+k} \\
& >k-1-\frac{1}{(k-1) n}-\frac{2}{(k-2) n}-\ldots-\frac{k-1}{n}>k-1-\frac{(k-1) H_{k-1}}{n} .
\end{aligned}
$$

Moreover, if $X_{i}^{\prime}=X_{(m+1-i n, m-i(n-1))}$ we have

$$
\sigma_{W_{n}}^{2}=\operatorname{Var}\left(W_{n}\right)=\sum_{i=1}^{k-1} \sum_{j=1}^{k-1} \operatorname{Cov}\left(X_{i}, X_{j}\right) \leq \sum_{i=1}^{k-1} \sum_{j=1}^{k-1} \sqrt{\operatorname{Var}\left(X_{i}\right) \operatorname{Var}\left(X_{j}\right)} \leq\left(\sum_{i=1}^{k-1} \sqrt{\operatorname{Var}\left(X_{i}\right)}\right)^{2}
$$




$$
<\left(\sum_{i=1}^{k-1} \frac{\sqrt{i}}{m-i n+i+1}\right)^{2}<\left(\sqrt{k-1} \sum_{i=1}^{k-1} \frac{1}{(k-i) n}\right)^{2}=\frac{(k-1) H_{k-1}^{2}}{n^{2}},
$$

where $H_{k-1}$ is the $(k-1)$-th harmonic number. Now we can bound the probability that any agent receives value less than $1 / n$ of her total value.

$$
\mathrm{P}\left(Y_{i}<\frac{v_{i}(M)}{n}\right) \leq \mathrm{P}\left(Y_{n}<\frac{v_{n}(M)}{n}\right) \leq \mathrm{P}\left(Y_{n}<\frac{13 k}{20}\right)+\mathrm{P}\left(\frac{v_{n}(M)}{n}>\frac{13 k}{20}\right) .
$$

Next, using Chebyshev's inequality we have

$$
\begin{aligned}
\mathrm{P}\left(Y_{n}<\frac{13 k}{20}\right) & \leq \mathrm{P}\left(W_{n}<\frac{13 k}{20}\right)=\mathrm{P}\left(\mathrm{E}\left[W_{n}\right]-W_{n}>\mathrm{E}\left[W_{n}\right]-\frac{13 k}{20}\right) \\
& \leq \mathrm{P}\left(\left|\mathrm{E}\left[W_{n}\right]-W_{n}\right|>k-1-\frac{(k-1) H_{k-1}}{n}-\frac{13 k}{20}\right) \\
& \leq \mathrm{P}\left(\left|\mathrm{E}\left[W_{n}\right]-W_{n}\right|>\frac{\frac{7 k}{20}-1-\frac{(k-1) H_{k-1}}{n}}{\frac{\sqrt{k-1} H_{k-1}}{n}} \sigma_{W_{n}}\right) \\
& \leq \frac{(k-1) H_{k-1}^{2}}{\left(\left(\frac{7 k-20}{20}\right) n-(k-1) H_{k-1}\right)^{2}} .
\end{aligned}
$$

On the other hand, using Hoeffding's inequality,

$$
\begin{aligned}
\mathrm{P}\left(\frac{v_{n}(M)}{n}>\frac{13 k}{20}\right) & =\mathrm{P}\left(\frac{v_{n}(M)}{m}-\frac{1}{2}>\frac{n}{m} \frac{13 k}{20}-\frac{1}{2}\right) \\
& \leq \mathrm{P}\left(\frac{v_{n}(M)}{m}-\frac{1}{2}>\frac{13 k}{20(k+1)}-\frac{1}{2}\right) \\
& \leq e^{-2 m\left(\frac{3 k-10}{20(k+1)}\right)^{2}} \leq e^{-2 k n\left(\frac{3 k-10}{20(k+1)}\right)^{2}} .
\end{aligned}
$$

Finally, we take a union bound to get

$$
\begin{aligned}
\mathrm{P}\left(\exists i \text { s.t. } Y_{i}<\frac{v_{i}(M)}{n}\right) & \leq \sum_{i=1}^{n} \mathrm{P}\left(Y_{i}<\frac{v_{i}(M)}{n}\right) \\
& \leq n\left(\frac{(k-1) H_{k-1}^{2}}{\left(\left(\frac{7 k-20}{20}\right) n-(k-1) H_{k-1}\right)^{2}}+e^{-2 k n\left(\frac{3 k-10}{20(k+1)}\right)^{2}}\right)=O(1 / n) .
\end{aligned}
$$

The exact same proof works when $3 n \leq m<3.5 n$, but instead of $\frac{3 k-10}{20(k+1)}$ in Hoeffding's inequality, we have $\frac{3 \cdot 3-5}{20(3+0.5)}$ and of course we should adjust $\mathrm{E}\left[W_{n}\right]$ and $\operatorname{Var}\left(W_{n}\right)$ accordingly. When $3.5 n \leq m<4 n$ on the other hand, we need to consider three items in $W_{n}$ instead of two, since two items are not enough anymore to guarantee separation of $Y_{i}$ and $\frac{1}{n} \sum_{j=1}^{m} v_{i j}$ with high probability. That said, the proof is the same, but we should adjust $\mathrm{E}\left[W_{n}\right]$ and $\operatorname{Var}\left(W_{n}\right)$, and instead of $\frac{13 k}{20}=\frac{39}{20}$ we may choose 2.5 .

We now state a similar result for any $m$, generalizing Theorem 6.2 that only holds when $m \geq 2 n$. We use a modification of Greedy Round-Robin. While $m<2 n$, the algorithm picks any agent uniformly at random and gives her only her "best" item (phase 1). When the number of available items becomes two times the number of active agents, the algorithm proceeds as usual (phase 2). We note that while for $m \geq 2 n$ Theorem 6.2 gives the stronger guarantee of $\frac{v_{i}(M)}{n}$ for each agent $i$, here we can only have a guarantee of $\boldsymbol{\mu}_{i}(n, M)$. 
THEOREM 6.3. Let $N=[n], M=[m]$, and the $v_{i j} s$ be as in Theorem 6.2. Then, for any $m$ and large enough $n$, the Modified Greedy Round-Robin algorithm allocates to each agent $i$ a set of items of total value at least $\boldsymbol{\mu}_{i}(n, M)$ with probability $1-o(1)$. The $o(1)$ term is $O(1 / n)$ when $m>2 n$ and $O(\log n / n)$ when $m \leq 2 n$.

PRoOF. If $m \geq 2 n$ then this is a corollary of Theorem 6.2. When $m<2 n$, then for any agent $i$ we have $\max _{j}\left\{v_{i j}\right\} \geq \boldsymbol{\mu}_{i}(n, M)$. So the first agent that receives only her most valuable item has total value at least $\boldsymbol{\mu}_{i}(n, M)$. If $N_{a}, M_{a}$ are the sets of remaining agents and items respectively, after several agents were assigned one item in phase 1 of the algorithm, then by Lemma 3.4, for any agent $i \in N_{a}$, we have $\boldsymbol{\mu}_{i}\left(\left|N_{a}\right|, M_{a}\right) \geq \boldsymbol{\mu}_{i}(n, M)$. If $\left|M_{a}\right|<2\left|N_{a}\right|$ it is also true that $\max _{j \in M_{a}} v_{i j} \geq \boldsymbol{\mu}_{i}\left(\left|N_{a}\right|, M_{a}\right)$, so correctness of phase 1 follows by induction. If $\left|M_{a}\right|=2\left|N_{a}\right|$, then by Theorem 6.2 phase 2 guarantees that with high probability each agent $i \in N_{a}$ will receive a set of items with total value at least $\frac{1}{\left|N_{a}\right|} v_{i}\left(M_{a}\right) \geq \boldsymbol{\mu}_{i}\left(\left|N_{a}\right|, M_{a}\right) \geq \boldsymbol{\mu}_{i}(n, M)$.

Remark 1. The implicit constants in the probability bounds of Theorems 6.2 and 6.3 depend heavily on $n$ and $m$, as well as on the point one uses to separate $Y_{i}$ and $\frac{1}{n} \sum_{j=1}^{m} v_{i j}$ in the proof of Theorem 6.2. Our analysis gives good bounds for the case $2 n \leq m<3 n$ without requiring very large values for $n$ (especially when $\kappa(n)$ in the proof of Theorem 6.2 is not small). For example, if $m=2.4 n$ an appropriate adjustment of our bounds gives a $o(1)$ term less than $1.7 / n$ for $n \geq 41$. When we switch from the detailed analysis of the $2 n \leq m<3 n$ case to the sloppier general treatment for $m \geq 3 n$, there is definitely some loss, e.g., for $m=4 n$ we get that the $o(1)$ term is less than $130 / n$ for $n>450$. This is corrected relatively quickly as $m$ grows, e.g., for $m=13 n$ the $o(1)$ term can be made less than $8 / n$ for $n \geq 59$. One can significantly improve the constants by breaking the interval $k n \leq m<(k+1) n$ into smaller intervals (not unlike the $3 n \leq m<3.5 n$ case).

Theorems 6.2 and 6.3 may leave the impression that $n$ has to be large. Actually, there is no reason why we cannot consider $n$ fixed and let $m$ grow. Following closely the proof of Theorem 6.2 for $m \geq 4 n$, we get the next corollary. Notice that now we can use $\mathrm{E}\left[W_{n}\right] \geq 0.7 k$ and $\sigma_{W_{n}}^{2}<k$.

Corollary 6.4. Let $N=[n], M=[m]$, and the $v_{i j}$ s be as in Theorem 6.2. Then, for fixed $n$ and large enough $m$, the Greedy Round-Robin algorithm allocates to each agent $i$ a set of goods of total value at least $\frac{1}{n} \sum_{j=1}^{m} v_{i j}$ with probability $1-O(1 / m)$.

\section{CONCLUSIONS}

The most interesting open question is undoubtedly whether one can improve on the $2 / 3$-approximation. Going beyond $2 / 3$ seems to require a drastically different approach. One idea that may deserve further exploration is to pick in each step of Algorithm 4, the best out of all possible matchings (and not just an arbitrary matching as is done in line 7 of the algorithm). This is essentially what we exploit for the special case of $n=3$ agents. However, for a larger number of agents, this seems to result in a heavy case analysis without any visible benefits. In terms of non-combinatorial techniques, we are not currently aware of any promising LP-based approach to the problem.

Even establishing better ratios for special cases could still provide new insights into the problem. It would be interesting, for example, to see if we can have an improved ratio for the special case studied in Bansal and Sviridenko [2006] for the Santa Claus problem. In this case of additive functions, the value of a good $j$ takes only two distinct values, 0 or $v_{j}$. On the other hand, obtaining negative results seems to be an even more challenging task, given our probabilistic analysis and the results of related works. The negative results [Kurokawa et al. 2016; Procaccia and Wang 2014] require 
very elaborate constructions, which still do not yield an inapproximability factor far away from 1. Apart from improving the approximation quality, exploring practical aspects of our algorithms is another direction, see e.g., Spliddit [2015]. Finally, we have not addressed here the issues of truthfulness and mechanism design, a stimulating topic for future work, studied recently by [Amanatidis et al. 2016; Amanatidis et al. 2017]. These works still leave several open questions regarding the approximability that can be achieved under truthfulness (without payments) for more than two agents. It is also not clear if more positive results can arise when payments are allowed. Similar mechanism design questions also remain open for a related problem studied by Markakis and Psomas [2011].

\section{REFERENCES}

G. Amanatidis, G. Birmpas, G. Christodoulou, and E. Markakis. 2017. Truthful Allocation Mechanisms Without Payments: Characterization and Implications on Fairness. In ACM Conference on Economics and Computation, EC'17. 545-562.

G. Amanatidis, G. Birmpas, and E. Markakis. 2016. On Truthful Mechanisms for Maximin Share Allocations. In Proceedings of the 25th International Joint Conference on Artificial Intelligence, IJCAI 2016. 31-37.

G. Amanatidis, E. Markakis, A. Nikzad, and A. Saberi. 2015. Approximation Algorithms for Computing Maximin Share Allocations. In Automata, Languages, and Programming - 42nd International Colloquium, ICALP 2015, Proceedings, Part I. 39-51.

A. Asadpour and A. Saberi. 2007. An approximation algorithm for max-min fair allocation of indivisible goods. In ACM Symposium on Theory of Computing (STOC). 114-121.

H. Aziz and S. MacKenzie. 2016. A Discrete and Bounded Envy-free Cake Cutting Protocol for Four Agents. In 48th ACM Symposium on the Theory of Computing, STOC 2016. 454-464.

N. Bansal and M. Sviridenko. 2006. The Santa Claus Problem. In ACM Symposium on Theory of Computing (STOC). 31-40.

S. Barman and S. K. K. Murthy. 2017. Approximation Algorithms for Maximin Fair Division. In ACM Conference on Economics and Computation, EC'17. 647-664.

I. Bezakova and V. Dani. 2005. Allocating Indivisible Goods. ACM SIGecom Exchanges 5 (2005), 11-18.

S. Bouveret, Y. Chevaleyre, and N. Maudet. 2016. Fair Allocation of Indivisible Goods. In Handbook of Computational Social Choice, F. Brandt, V. Conitzer, U. Endriss, J. Lang, and A. D. Procaccia (Eds.). Cambridge University Press, Chapter 12.

S. Bouveret and J. Lang. 2011. A General Elicitation-free Protocol for Allocating Indivisible Goods. In Proceedings of the 22nd International Joint Conference on Artificial Intelligence, IJCAI 2011. 73-78.

S. Bouveret and M. Lemaître. 2016. Characterizing conflicts in fair division of indivisible goods using a scale of criteria. Autonomous Agents and Multi-Agent Systems 30, 2 (2016), 259-290. A preliminary version of this work has appeared in AAMAS '14.

S. J. Brams and D. King. 2005. Efficient Fair Division - Help the worst off or avoid envy. Rationality and Society 17, 4 (2005), 387-421.

S. J. Brams and A. D. Taylor. 1996. Fair Division: from Cake Cutting to Dispute Resolution. Cambridge University press.

E. Budish. 2011. The Combinatorial Assignment Problem: Approximate Competitive Equilibrium from Equal Incomes. Journal of Political Economy 119, 6 (2011), 1061-1103.

I. Caragiannis, D. Kurokawa, H. Moulin, A. D. Procaccia, N. Shah, and J. Wang. 2016. The Unreasonable Fairness of Maximum Nash Welfare. In ACM Conference on Economics and Computation, EC '16. 305322.

J. Edmonds and K. Pruhs. 2006. Balanced Allocations of Cake. In Symposium on Foundations of Computer Science (FOCS). 623-634.

S. Even and A. Paz. 1984. A note on cake cutting. Discrete Applied Mathematics 7 (1984), 285-296.

J.E. Gentle. 2009. Computational statistics. Vol. 308. Springer.

P. Hall. 1935. On Representatives of Subsets. Journal of the London Mathematical Society s1-10, 1 (1935), 26-30.

W. Hoeffding. 1963. Probability Inequalities for Sums of Bounded Random Variables. J. Amer. Statist. Assoc. 58, 301 (1963), 13-30. 
D. Kurokawa, A. D. Procaccia, and J. Wang. 2016. When Can the Maximin Share Guarantee Be Guaranteed?. In 30th AAAI Conference on Artificial Intelligence, AAAI 2016.

E. Markakis and C.-A. Psomas. 2011. On Worst-Case Allocations in the Presence of Indivisible Goods. In $7 t h$ Workshop on Internet and Network Economics (WINE 2011). 278-289.

H. Moulin. 1990. Uniform Externalities: Two Axioms for Fair Allocation. Journal of Public Economics 43, 3 (1990), 305-326.

A. D. Procaccia. 2015. Cake Cutting Algorithms. In Handbook of Computational Social Choice, F. Brandt, V. Conitzer, U. Endriss, J. Lang, and A.D. Procaccia (Eds.). Cambridge University Press, Chapter 13.

A. D. Procaccia and J. Wang. 2014. Fair enough: guaranteeing approximate maximin shares. In ACM Conference on Economics and Computation, EC '14. 675-692.

J. M. Robertson and W. A. Webb. 1998. Cake Cutting Algorithms: be fair if you can. AK Peters.

Spliddit. 2015. Provably Fair Solutions. http://www.spliddit.org/. (2015).

H. Steinhaus. 1948. The Problem of Fair Division. Econometrica 16 (1948), 101-104.

G. Woeginger. 1997. A polynomial time approximation scheme for maximizing the minimum machine completion time. Operations Research Letters 20 (1997), 149-154.

G. Woeginger and J. Sgall. 2007. On the Complexity of cake cutting. Discrete Optimization 4, 2 (2007), 213220. 